\title{
Global regulatory features of alternative splicing across tissues and within the nervous system of $C$. elegans
}

\author{
Bina Koterniak, ${ }^{1,5}$ Pallavi P. Pilaka, ${ }^{1,5}$ Xicotencatl Gracida, ${ }^{2,5}$ Lisa-Marie Schneider, ${ }^{1,3}$ \\ Iva Pritišanac, ${ }^{1,4}$ Yun Zhang, ${ }^{2}$ and John A. Calarco ${ }^{1}$ \\ ${ }^{1}$ Department of Cell and Systems Biology, University of Toronto, Toronto, Ontario M5S 3G5, Canada; ${ }^{2}$ Department of Organismal and \\ Evolutionary Biology, Harvard University, Cambridge, Massachusetts 02138, USA; ${ }^{3}$ Department of Chemistry, University of Bayreuth, \\ 95447 Bayreuth, Germany; ${ }^{4}$ Program in Molecular Medicine, The Hospital for Sick Children, Toronto, Ontario M5G 0A4, Canada
}

\begin{abstract}
Alternative splicing plays a major role in shaping tissue-specific transcriptomes. Among the broad tissue types present in metazoans, the central nervous system contains some of the highest levels of alternative splicing. Although many documented examples of splicing differences between broad tissue types exist, there remains much to be understood about the splicing factors and the cis sequence elements controlling tissue and neuron subtype-specific splicing patterns. By using translating ribosome affinity purification coupled with deep-sequencing (TRAP-seq) in Caenorhabditis elegans, we have obtained high coverage profiles of ribosome-associated mRNA for three broad tissue classes (nervous system, muscle, and intestine) and two neuronal subtypes (dopaminergic and serotonergic neurons). We have identified hundreds of splice junctions that exhibit distinct splicing patterns between tissue types or within the nervous system. Alternative splicing events differentially regulated between tissues are more often frame-preserving, are more highly conserved across Caenorhabditis species, and are enriched in specific cis regulatory motifs, when compared with other types of exons. By using this information, we have identified a likely mechanism of splicing repression by the RNA-binding protein UNC-75/CELF via interactions with cis elements that overlap a $5^{\prime}$ splice site. Alternatively spliced exons also overlap more frequently with intrinsically disordered peptide regions than constitutive exons. Moreover, regulated exons are often shorter than constitutive exons but are flanked by longer intron sequences. Among these tissue-regulated exons are several highly conserved microexons $<27 \mathrm{nt}$ in length. Collectively, our results indicate a rich layer of tissue-specific gene regulation at the level of alternative splicing in $C$. elegans that parallels the evolutionary forces and constraints observed across metazoa.
\end{abstract}

[Supplemental material is available for this article.]

Alternative precursor mRNA (pre-mRNA) splicing is a critical layer of gene expression in multicellular animals and plays a key role in increasing the functional diversity of proteins within an organism. Indeed, a significant fraction of multiexon genes undergo alternative splicing, with higher frequencies reported across vertebrates (Pan et al. 2008; Wang et al. 2008; Barbosa-Morais et al. 2012; Merkin et al. 2012) in comparison to invertebrate model organisms such as the nematode Caenorhabditis elegans and the fruit fly Drosophila melanogaster (Gerstein et al. 2010; Brown et al. 2014). Splice isoforms are often differentially expressed across tissues and/or during development (for select examples, see Pan et al. 2008; Wang et al. 2008; Gerstein et al. 2010; Ramani et al. 2011; Brown et al. 2014). As such, it remains an important goal to identify the repertoire of splice variants that help define cell- and tissue-specific functions in multicellular animals.

The relevance of alternative splicing in establishing proteomic diversity is especially reflected in the nervous system. Given its cellular complexity, the nervous system displays elevated levels of tissue-specific splicing that are also conserved throughout vertebrate evolution (Xu et al. 2002; Yeo et al.

\footnotetext{
${ }^{5}$ These authors contributed equally to this work. Corresponding author: john.calarco@utoronto.ca

Article published online before print. Article, supplemental material, and publication date are at http://www.genome.org/cgi/doi/10.1101/gr.267328.120. Freely available online through the Genome Research Open Access option.
}

2004; Barbosa-Morais et al. 2012; Merkin et al. 2012). More recently, transcriptome profiling in specific neuronal subtypes has revealed an even greater degree of transcript diversity generated by alternative splicing (Wamsley et al. 2018; Wang et al. 2018; Furlanis et al. 2019; Saito et al. 2019). Alternative splicing in the nervous system regulates an array of physiological events and properties, such as neurogenesis, neuronal migration, synaptic plasticity, and ion channel kinetics, and contributes to the diversity needed to fine-tune the exact activity of neurons (Ule and Darnell 2006; Norris and Calarco 2012; Raj and Blencowe 2015; Vuong et al. 2016). Alternative splicing must be tightly regulated, especially in coding sequences, to give rise to functional proteins. Splice site selection and tissue/developmental specificity are established by auxiliary trans-acting proteins and cis-acting sequence elements, which are primarily RNA-binding proteins (RBPs) and their cognate motifs, respectively (Lee and Rio 2015). Collectively, these elements direct the assembly and activity of the spliceosome and are often referred to as the "splicing code" (Wang and Burge 2008).

Although significant progress has been made in understanding the combinations of features enabling the accurate prediction of splicing outcomes (Barash et al. 2010; Zhang et al. 2010; Xiong

(C) 2020 Koterniak et al. This article, published in Genome Research, is available under a Creative Commons License (Attribution 4.0 International), as described at http://creativecommons.org/licenses/by/4.0/. 
Tissue-specific alternative splicing in C. elegans

et al. 2015; Baeza-Centurion et al. 2019; Jaganathan et al. 2019), our understanding of the splicing code remains incomplete. This fact is not surprising given that alternative splicing is regulated in a combinatorial manner, with multiple cis elements and/or multiple RBPs cooperating or antagonizing each other to generate splicing outcomes (Fu and Ares 2014). Additionally, the inclusion of tissue, cell, and developmental contexts is required in order to accurately predict splicing outcomes, as a number of RBPs vary correspondingly in expression patterns and as the majority of alternative splicing events are tissue and developmentally regulated (Gerstberger et al. 2014).

The nematode worm C.elegans possesses multiple tissue types, including a well-differentiated nervous system estimated to contain at least 118 distinct neuronal subtypes, including the major neurotransmitter-releasing classes found in vertebrates (White et al. 1986; Hobert 2010). Moreover, $C$. elegans has served as an excellent model system to study the mechanisms governing alternative splicing regulation (Zahler 2012; Gracida et al. 2016; Wani and Kuroyanagi 2017). Recent investigations harnessing the tractability of this organism have focused on whole-animal or tissue-enriched transcriptome sequencing approaches to explore changes in posttranscriptional gene regulation during development and aging (Barberan-Soler and Zahler 2008; Barberan-Soler et al. 2009; Gerstein et al. 2010; Ramani et al. 2011; Blazie et al. 2015, 2017; Ragle et al. 2015; Kaletsky et al. 2018; Kotagama et al. 2019; Warner et al. 2019; Li et al. 2020; Roach et al. 2020). However, it still remains unclear how splicing in C. elegans is regulated at the level of tissues, in particular at the resolution of specific neuronal subtypes. A better understanding of these mechanisms will require the systematic search for features of alternative splicing events, exploration of the evolutionary dynamics of splice isoform usage, and tools for the unbiased identification of splicing regulators.

To address these questions, we performed a genome-wide analysis of alternative splicing in C. elegans using broad tissue-specific and neuronal subtypespecific ribosome-associated mRNAs obtained by the translating ribosome affinity purification (TRAP) approach coupled with deep-sequencing (TRAP-seq) (Gracida and Calarco 2017). By using these data, we sought to determine the extent to which TRAP enriched tissuespecific and neuron subtype-specific splice junctions are undetected in whole-animal data. Additionally, we aimed to examine these events for distinct sequence and conservation features that may delineate mechanisms of splicing regulation.

\section{Results}

Tissue-enriched transcript data identifies splice junctions not identified in whole-animal profiling data

We have successfully adapted the TRAP technique (Heiman et al. 2008) for use in C. elegans and coupled it with deep-sequencing (TRAP-seq) (Gracida and Calarco 2017). In this technique, a green fluorescent protein (GFP) tagged ribosomal subunit protein, RPL1 , is expressed under the control of tissue- or cell-specific promoters (Fig. 1A). Whole-animal lysates are then prepared in the presence of cycloheximide, arresting ribosomes on mRNAs that are being actively translated. Ribosomes and their associated mRNAs are then immunoprecipitated from the lysate via the GFP tag, thus enriching for transcripts present in the original cell types expressing the GFP-tagged ribosomal protein. We used TRAP-seq to
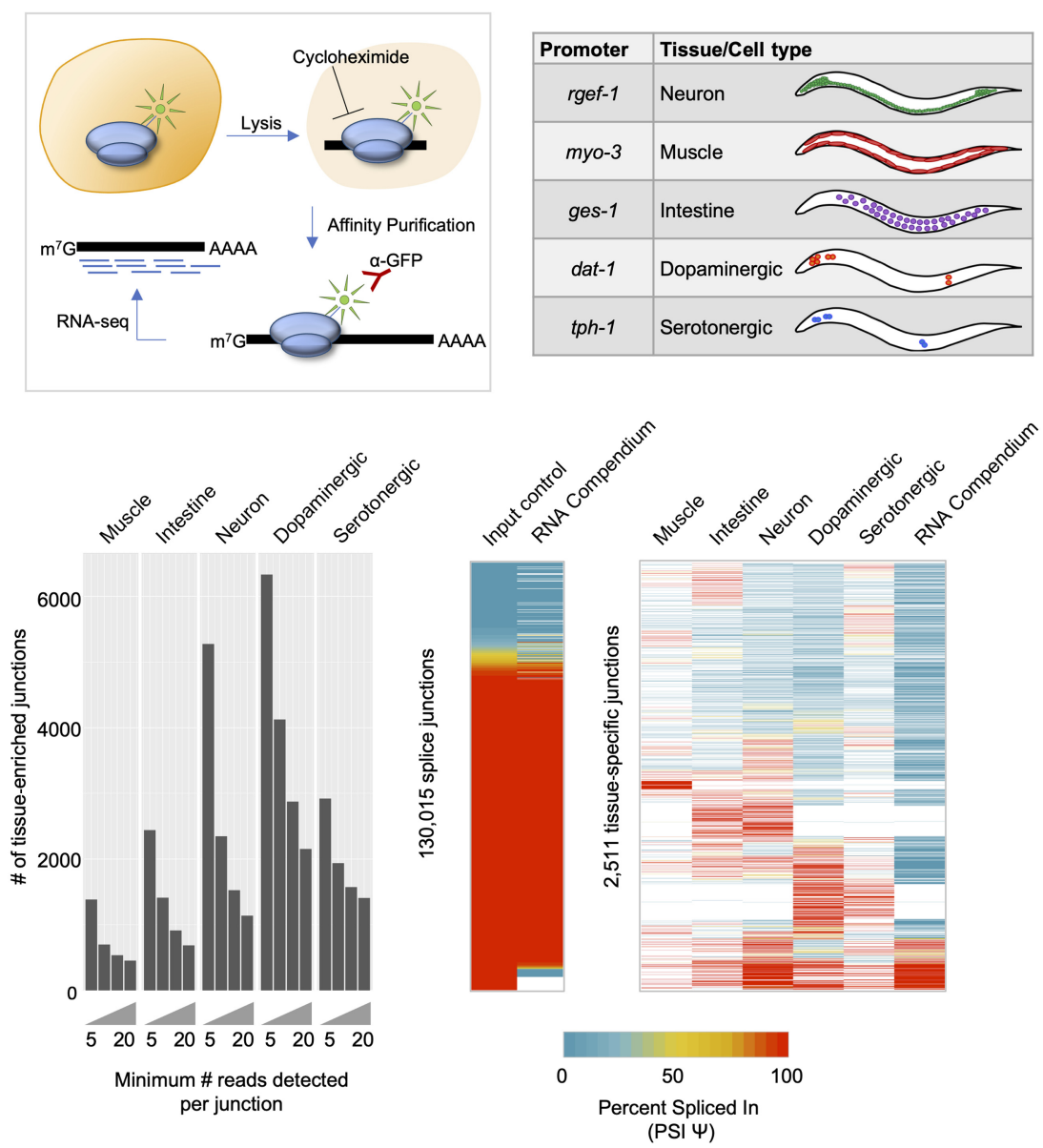

Figure 1. TRAP-seq identifies tissue-enriched junctions not found in whole-animal data. $(A)$ Experimental outline for TRAP-seq approach. Animals expressing a GFP-tagged ribosomal protein are lysed in the presence of cycloheximide, and lysates are immunoprecipitated with anti-GFP antibodies, enriching for ribosome-associated mRNAs. cDNA libraries are prepared and then sequenced. (B) TRAP strains used in the present study, with reference gene listed from where promoters were cloned, as well as broad tissues or neuron subtypes for which mRNA profiles were obtained using TRAP-seq. ( $C$ ) Number of tissuespecific splice junctions from each TRAP-seq IP sample that are absent from our whole-animal input control transcriptomes over increasing read count support thresholds. $(D$, left) Comparison of our whole-animal input control transcriptome data and RNA compendium data curated by Tourasse et al. (2017). PSI values for all input junctions and corresponding RNA compendium junctions are shown in the heatmap. Pearson correlation coefficient, $\mathrm{R}=0.91, P<2.2 \times 10^{-16}$. (Right) PSI values of alternatively spliced tissuespecific junctions with at least 20 supporting reads, and corresponding PSI values in the RNA compendium. White indicates cases in which no reads were detected in a particular sample. 
obtain high coverage mRNA repertoire measurements from C. elegans fourth-larval-stage (L4) animals for three broad tissue classes -neurons, muscle, and intestine-and two neuronal subtypesdopaminergic and serotonergic neurons (Fig. 1B). Two biological replicates of TRAP-enriched mRNA and matched whole-animal mRNA (represented by mRNA purified from our lysates before immunoprecipitation) were collected from each transgenic strain. In total, over 2 billion uniquely mapped reads were aligned across all data sets, with summed coverage for each tissue-enriched or cell type-enriched transcriptome ranging from over 100 million to over 300 million reads (Supplemental Fig. S1).

We first mapped our TRAP-seq reads to the C. elegans genome and to existing gene models (for details, see Methods) and identified all aligned reads supporting annotated splice junctions, as well as high-confidence unannotated splice junctions. In total, we detected 145,811 splice junctions supported by at least five read counts across all samples (Supplemental Table S1). We next investigated whether our tissue-enriched mRNA populations enabled the detection of splice junctions that are absent owing to dilution in our whole-animal transcriptome data. In each tissue or cell type, we detected between approximately 1400 and over 6000 splice junctions supported by at least five reads that were absent from our whole-animal transcriptome data sets (Fig. 1C). Even when requiring that tissue-enriched splice junctions be supported by more than 20 reads, we still identified a total of 5577 nonredundant splice junctions not detected in our whole-animal data, spanning 1396 genes (Fig. 1C).

We next asked whether our tissue-enriched splice junctions were detected in whole-animal samples upon pooling a larger number of transcriptome data sets. To perform this analysis, we used a previously published RNA-seq compendium of reproducibly expressed splice junctions in C. elegans, assembled from 1682 publicly available RNA-seq experiments (Tourasse et al. 2017). Each splice junction has an associated percent spliced in (PSI) value, which is a measure of the frequency of each splice junction relative to other junctions sharing the same splice donor or acceptor sites (for details, see Methods). This schema for calculating the PSI value was applied to our data in order to compare our measurements with the RNA-seq compendium.

Both our whole-animal splice junction PSI value measurements and those of the RNA-seq compendium were strongly correlated, despite being derived from different data sets $(r=0.91$, Pearson correlation coefficient) (Fig. 1D; left panel). However, when comparing our TRAP-seq tissue-enriched and cell type-enriched splice junctions supported by at least 20 counts with the RNA-seq compendium, we observed that $\sim 45 \%$ of these splice junctions are not detected in the RNA-seq compendium (Fig. 1D; right panel). We also asked whether the tissue-enriched splice junctions show distinct splicing patterns across tissues. We detected 2511 splice junctions with evidence of alternative splicing (where a minor splice variant must have a PSI value $\geq 5 \%$ ) (Fig. $1 \mathrm{D}$; right panel). Many of these splice junctions are not rare variants owing to their high PSI values and also display differentially regulated splicing patterns across tissues (Fig. 1D; right panel). Taken together, these results show that our TRAP-seq tissue-enriched mRNA profiles reveal splice junctions that are difficult to detect in whole-animal transcriptomes.

\section{Independent validation of TRAP-seq measurements using in vivo two-color splicing reporters}

We next sought to validate our TRAP-seq measurements through the use of an independent approach. We used a new two-color fluorescent splicing reporter based on previous design strategies (Fig. 2A; Kuroyanagi et al. 2010; Norris et al. 2014). Specifically, these two-color reporters are designed to include an alternative exon of interest and its flanking introns and exons (referred to as a minigene). Upstream of the minigene is the promoter that drives the tissue-specific expression of the fluorescent markers. Downstream from the minigene, we inserted sequences encoding enhanced GFP (EGFP) or the red fluorescent protein mCherry, each translated in separate reading frames. The alternative exon in the minigene is then engineered to switch between both reading frames, such that when the alternative exon is skipped, EGFP is translated, and, when the exon is included, mCherry protein is produced (Fig. 2A). A similar frame-shifting strategy is applied to monitor alternative $5^{\prime}$ and $3^{\prime}$ splice site selection events (Supplemental Fig. S2A).

Additionally, our reporter included 2A peptide sequences upstream of each fluorescent protein to cleave away upstream peptides translated from the minigene exons (Ahier and Jarriault 2014). Finally, two nuclear localization signals (SV40 and EGL13) (Lyssenko et al. 2007) were included at the $\mathrm{N}$ and $\mathrm{C}$ termini of each fluorescent protein to concentrate signal in the nucleus of each cell expressing the reporter. With this combined set of design features, these reporters enable the visualization of alternative splicing patterns by fluorescence microscopy in vivo and at singlecell resolution.

Our TRAP-seq data set was analyzed for splicing events that showed switch-like splicing patterns (a change in PSI value, or $\Delta$ PSI, of $\geq 80 \%$ ) between the broad tissue types. Four such events were cloned into our two-color reporters (minigene sequences surrounding $h l b-1$ exon 12, fhod-1 exon 8, zoo-1 exon 9, and ampd-1 exon 15), and expression of these reporters was driven under the control of neuronal, body-wall muscle, or intestinal promoters (for details, see Methods) (Fig. 2B,C; Supplemental Fig. S2; see Fig. 7A). In all cases tested, we observed agreement between fluorescent reporter patterns in vivo and our TRAP-seq measurements across tissues (Fig. 2B,C; Supplemental Fig. S2). We additionally monitored the splicing patterns of exon 7 in the $a d r-1$ gene and exon 11 in $m l k-1$. These splicing events were identified by TRAPseq as differentially regulated between serotonergic and dopaminergic neurons or between the neuronal subtypes and the rest of the nervous system, respectively. Again, our fluorescent reporter signals agreed with the TRAP-seq measurements (Fig. 2D; Supplemental Fig. S2). Including these reporters, a total of 14 two-color reporters were tested, and 11 reporters displayed patterns consistent with TRAP-seq measurements (Supplemental Table S2).

Taken together, although our TRAP-seq analysis and two-color reporters assess splicing patterns through different means, their general correlation strongly suggests that our TRAP-seq measurements can detect regulated splicing patterns.

\section{TRAP-seq identifies tissue-biased and neuronal subtype-biased splicing patterns}

We next used the MAJIQ software package (Vaquero-Garcia et al. 2016) to identify all the alternative splicing events (defined by MAJIQ as local splicing variation) in each TRAP-seq tissue transcriptome and all the tissue-differential splicing events within every pair of broad tissue types (muscle, intestine, neurons) and within the nervous system (neurons, dopaminergic neurons, serotonergic neurons; for details, see Methods) (Fig. 3). In total, we identified 2953 alternative splicing events in 2196 genes across all tissues. These alternative splicing events are distributed across

\section{Genome Research}

www.genome.org 
A

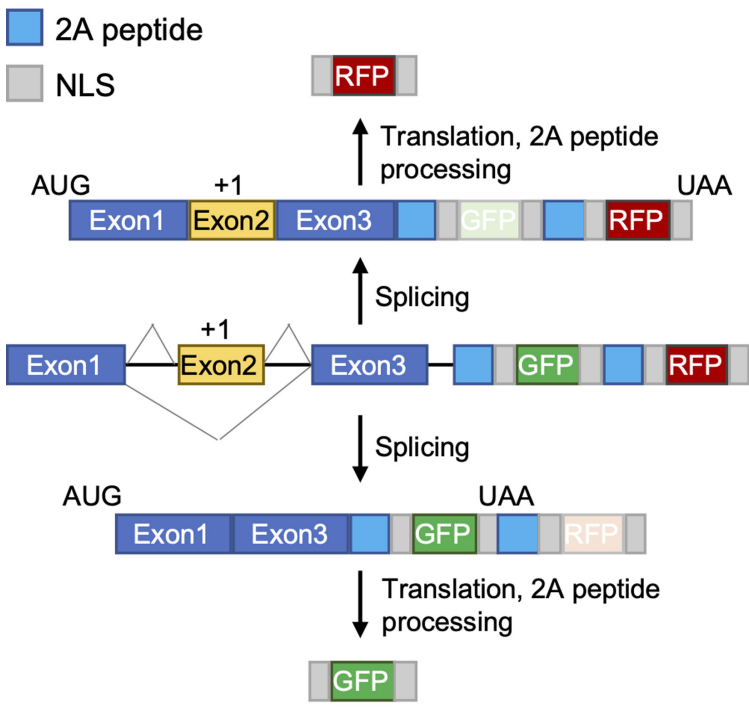

C
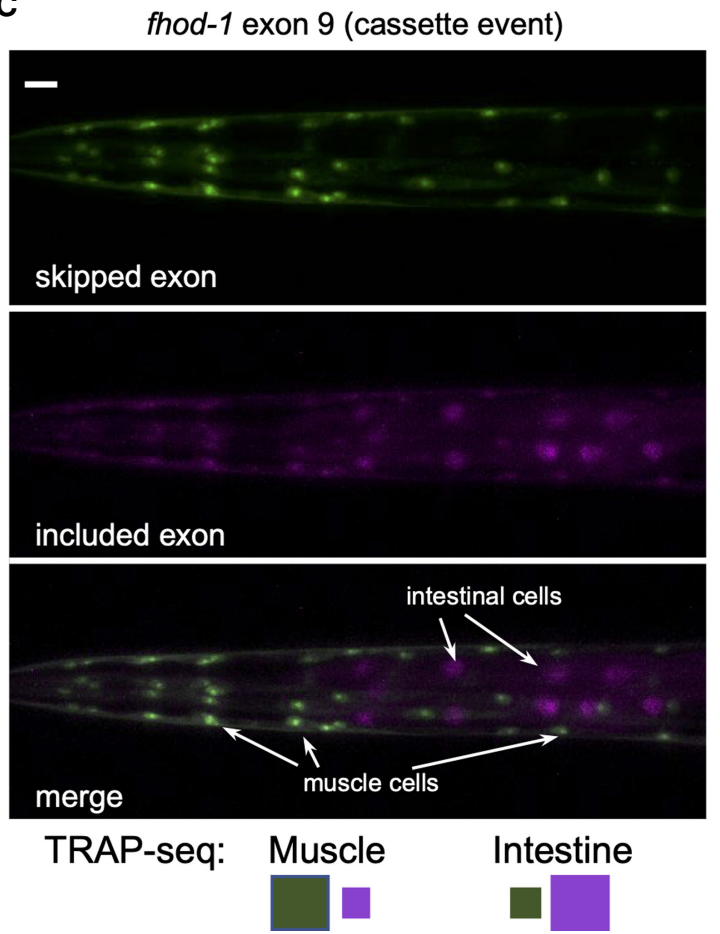

B hlb-1 exon 12 (alt. 5' splice site)
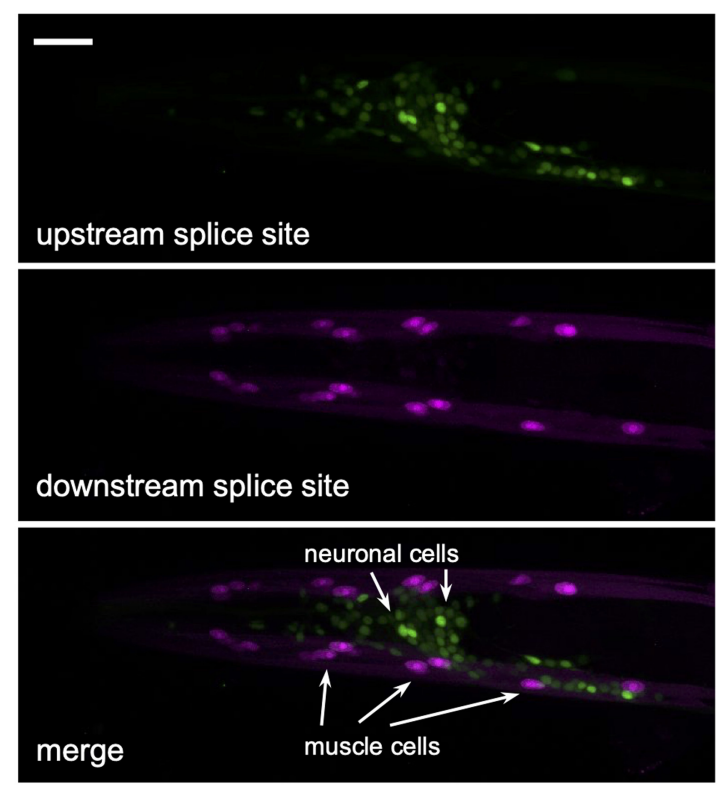

TRAP-seq: Neuron Muscle

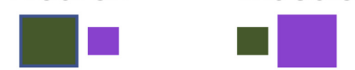

D
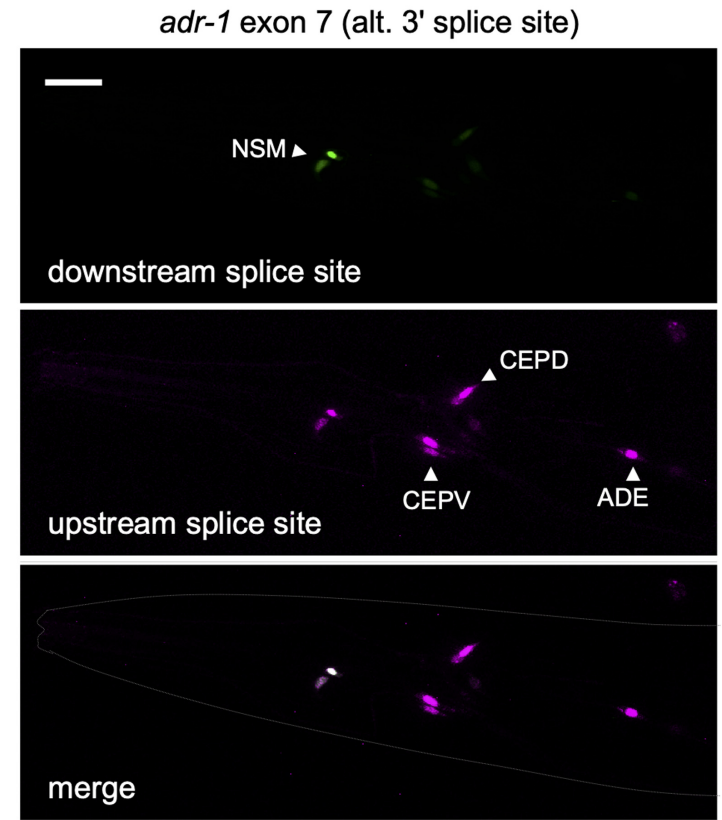

TRAP-seq: Serotonergic Dopaminergic

Figure 2. Validation of TRAP-seq measurements with two-color splicing reporters. $(A)$ Schematic of the two-color splicing reporter architecture. A minigene, comprising an alternatively spliced cassette exon (yellow) with flanking introns and flanking constitutive exons (blue), is cloned upstream of GFP and mCherry (RFP) ORFs. The alternative exon is engineered to include an extra nucleotide (+1) to shift reading frame. The ORFs differ in reading frame resulting in the expression of RFP upon inclusion of the cassette exon, whereas skipping of the exon leads to the expression of GFP. Cleavage at the 2A peptides and nuclear localization sequences (NLSs) ultimately leads to free fluorescent reporter proteins to accumulate in the nucleus. ( $B$ ) Fluorescence microscopy of splicing reporter expressed in neurons and muscle cells monitoring a splicing event in the $h / b-1$ gene. Top labels indicate gene, alternatively spliced exon, and splicing class; bottom labels indicate predominant color expected in reporter for a particular tissue (larger-size green or purple square) from measured PSI values from TRAP-seq data. Scale bar, $20 \mu \mathrm{m}$. (C) Same as in $B$, with splicing reporter expressed in intestine and muscle cells monitoring splicing event in fhod-1 gene. Scale bar, $20 \mu \mathrm{m}$. (D) Same as in B and C, with splicing reporter expressed in dopaminergic (CEP and ADE) and serotonergic (NSM) neurons monitoring splicing event in adr-1 gene. Scale bar, $20 \mu \mathrm{m}$. 
five major classes: cassette type (exon skipping), alternative $3^{\prime}$ or $5^{\prime}$ splice site usage, alternative start or terminal exons, intron retention, and mutually exclusive splicing (Fig. 3A; Supplemental Fig. S3; Supplemental Table S3). We also identified splicing events that we classified as "complex" (Fig. 3A; Supplemental Fig. S3). This latter class represents splice junctions making use of more than one mode of splicing, as well as accounts for $\sim 10 \%-15 \%$ of all forms of alternative splicing in a particular tissue or cell type. As described previously for whole-animal transcriptome data (Ramani et al. 2011), the predominant class of alternative splicing in any given tissue in C. elegans is alternative $3^{\prime}$ or $5^{\prime}$ splice site usage (Fig. 3A).

Moreover, our analysis of tissue-biased alternative splicing identified 871 differentially regulated splicing events in 689 genes across tissues and the two neuronal subtypes (Fig. 3B; Supplemental Table S4). Again, all major classes of splicing were represented among the set of differentially regulated alternative splicing events (Fig. 3B; Supplemental Table S3). However, complex splicing events represent a much larger proportion of tissuedifferential splicing compared with the distribution of possible splicing modes in a given tissue or cell type (Fig. 3, cf. A and B). For example, in the three broad tissue pairwise comparisons, complex splicing events accounted for between $40 \%$ and 50\% of all differentially regulated splicing events, a significantly increased proportion compared with individual cell or tissue types ( $P$ values all $<1 \times 10^{-4}$, chisquared test) (Fig. 3, cf. A and B).

Gene Ontology (GO) overrepresentation analysis was performed on the sets of genes with exons that were differentially spliced between any two tissues. These genes with differentially spliced exons were compared against a background set of genes expressed in the same tissues (see Methods). Splicing events differentially regulated between muscle, intestine, and neurons were enriched in genes involved in actin/cytoskeleton organization, muscle cell differentiation, neuronal differentiation, and the regulation of neuronal projections (Supplemental Table S5), similar to previous findings in vertebrates (Zheng and Black 2013; Giudice et al. 2014; Raj and Blencowe 2015).

Finally, we performed clustering analysis and quantification of noncomplex, differentially regulated splicing events expressed across at least two tissues and/or cell types (Fig. 3C-E). Our analysis revealed that fewer differences in splicing were found between muscle and intestinal cells compared with differences between these two tissues and the nervous system (Fig. 3D). Similarly, fewer differences were detected between the dopaminergic and serotonergic neurons, but we identified many more differences between these neuromodulatory cells and the broad nervous system samples (Fig. 3D). Accordingly, splicing patterns were correlated between muscle and intestinal cells $(r=0.63)$ and between dopaminergic and serotonergic neurons $(r=0.65)$, but very little correlation was observed between these tissues and neuron subtypes with the broad nervous system ( $r$ from -0.04 to 0.07 ) (Fig. 3E). 
Taken together, these results indicate that tissue-regulated splicing represents a substantial and complex layer of gene expression in C. elegans. Moreover, regulated splicing plays a particularly important role in shaping the transcriptome of the nervous system, including the fine-tuning of transcript variants expressed in dopaminergic and serotonergic neurons.

\section{Expression level differences and splicing pattern differences between tissues involve largely distinct sets of genes}

Splicing differences and steady-state mRNA differences between cell and tissue types are thought to have evolved on largely distinct sets of genes (Pan et al. 2004). To test this possibility in our tissuespecific transcriptome data, we performed a reciprocal clustering analysis of genes with differential expression or splicing patterns (Fig. 4A,B).

First, we identified all genes that were both differentially expressed between pairs of tissues and contain one or more alternative splicing events (13\% of all differentially expressed genes; see Methods). After clustering genes by their differential expression patterns, we observed no obvious trends with splicing differences in the same genes (Spearman's rho values between -0.01 and 0.04 , $P>0.3$ ) (Fig. 4A). Similarly, genes with tissue-differential splicing events were clustered according to their PSI value (Fig. 4B). Again, there was no obvious correlation between splicing patterns and steady-
A

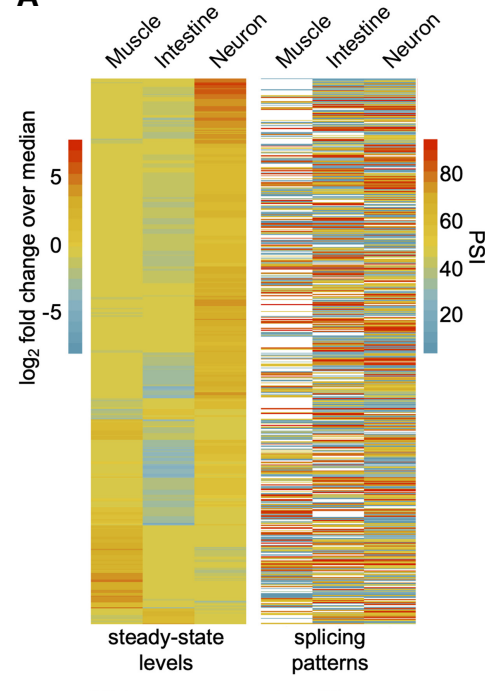

GO terms - expression differences

C

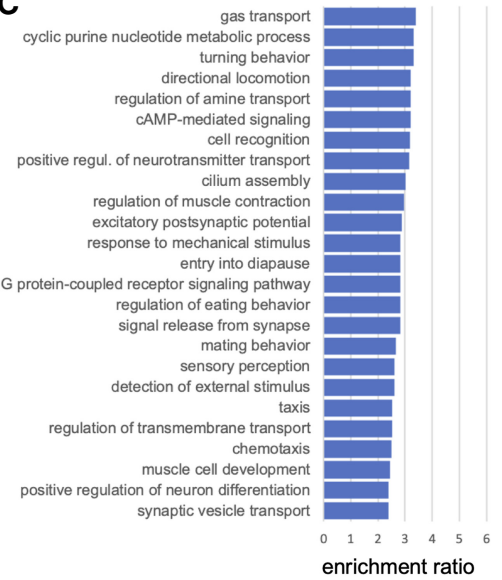

B

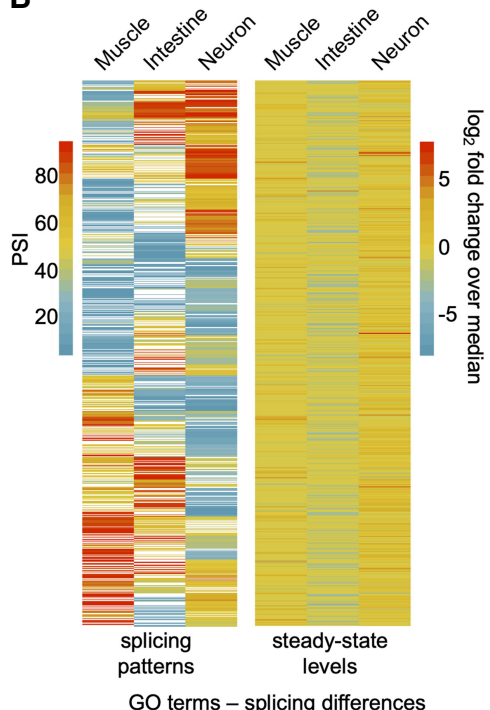

D

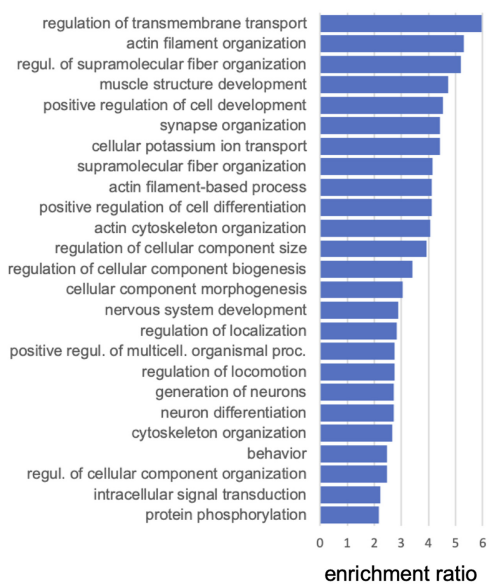

Figure 4. Tissue-regulated splicing and steady-state level differences are enriched in nonoverlapping gene sets. $(A$, left) Genes with significant steady-state transcript level differences clustered by their $\log _{2}$-transformed fold change over median ratios. (Right) PSI value measurements for alternative splicing events in the same genes ordered according to panel on the left. ( $B$, left) Genes with tissue-regulated splicing patterns clustered by their respective PSI value measurements. (Right) The $\log _{2}$-transformed fold change over median ratios for the same genes with splicing differences, ordered according to panel on the left. (C) Gene Ontology (GO) enrichment analysis of genes with steady-state transcript level differences from heatmap in $A$. Overrepresentation analysis was performed applying Bonferroni correction for multiple testing. Significantly enriched biological processes with corrected $P$-values $<0.05$ are shown. The enrichment ratio is the proportion of the number of genes in the sample set versus the number of genes in the background set for each GO category. Top $25 \mathrm{GO}$ terms are listed. Full list is available in Supplemental Table S6. (D) GO enrichment analysis of genes with tissue-regulated splicing events from heatmap in $B$. Analysis is as described in $C$. state RNA level differences (Spearman's rho values between -0.04 and -0.01 , $P>0.3$ ) (Fig. 4B). We observed similar patterns in comparisons between the nervous system and neuronal subtypes (Supplemental Fig. S4).

Consistent with these analyses above, the top overrepresented GO categories for genes with tissue-differential splicing patterns and genes with tissuedifferential expression levels were somewhat distinct (Fig. 4C,D). A wide range of biological processes are enriched in differentially expressed genes within the broad tissues, whereas differentially spliced genes are enriched in vesicle transport, actin kinetics, ion channel activity, and muscle/neuron differentiation (Fig. 4C,D; Supplemental Table S6). Taken together, our results indicate that regulated alternative splicing has contributed to tissue and cell type diversification by influencing a distinct set of genes compared with those regulated at the level of steady-state abundance.

\section{Tissue-regulated exons and their surrounding sequences are highly conserved across Caenorhabditis species}

Previous studies of tissue-regulated exons in vertebrates have shown that sequences flanking these exons tend to be more highly conserved (Sugnet et al. 2006; Fagnani et al. 2007). Thus, we next assessed whether tissue-regulated exons and their flanking introns and exons (exon triplets) were more likely to be conserved across the phylogeny of several Caenorhabditis species (Fig. 5A). For this analysis, we focused on comparing all cassette-type tissue-regulated exons with two other groups of exon triplets (composed of three exons and two introns). One group consisted of constitutively spliced events, in which three sequential exons are always spliced into mRNA transcripts. The 
A

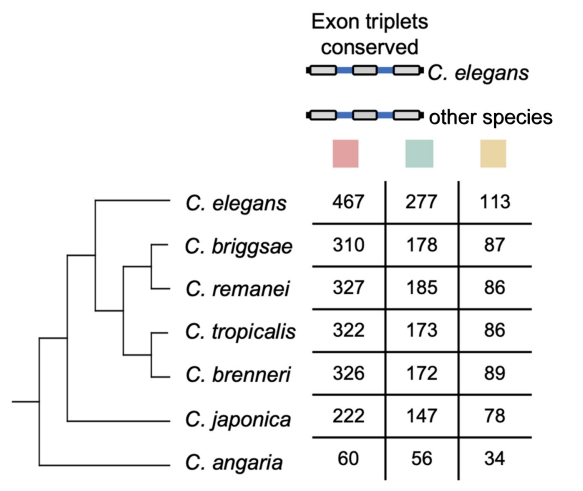

Constitutive exons Non-tissue-regulated alternative exons
B

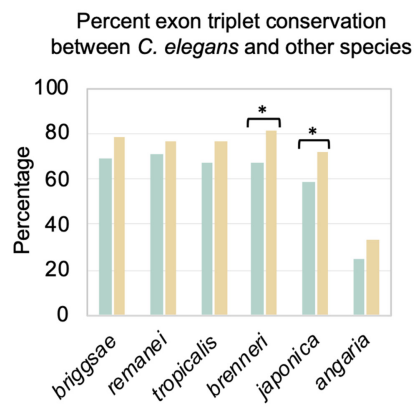

Tissue-regulated alternative exons

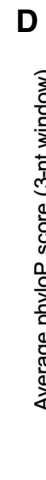

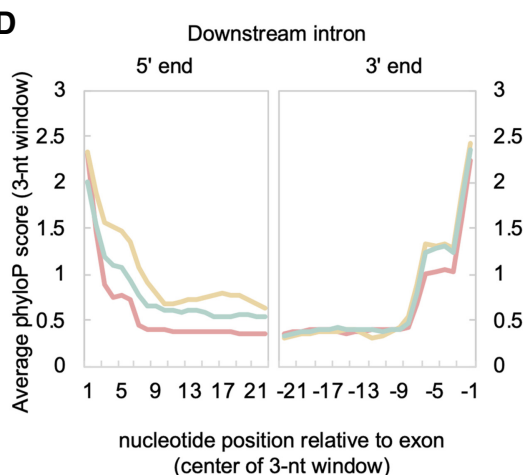

(center of 3-nt window)
Constitutive exons Non-tissue-regulated alternative exons

Tissue-regulated alternative exons

Figure 5. Alternative and tissue-regulated splicing are associated with increased conservation patterns. ( $A$, left) Phylogenetic tree of Caenorhabditis species used in alignments. (Right) Splicing events were separated into three classes of exon triplets: constitutive exons, non-tissue-regulated alternative exons, and tissue-regulated alternative exons. Table shows number of exon triplets in each group and their conservation across the phylogeny. $(B)$ Graph of percentage of conserved exon triplets for non-tissue-differential alternative exons (light blue bars) and tissue-differential alternative exons (gold bars) between C. elegans and other Caenorhabditis species of varying divergence. (*) P-value $<0.02$, Fisher's exact test. (C) Plot of average phyloP score (rolling 3-nt average) over first $23 \mathrm{nt}$ (left) and last $23 \mathrm{nt}$ (right) of the upstream intron flanking the internal exon of the exon triplets described in panel $A$. (D) Same as in $C$, but plots of the average phyloP score for the downstream intronic regions.

homologous sequence alignments over any part of their coding region (see Methods). Extending earlier comparisons with C. briggsae and C. remanei using EST/ cDNA data (Irimia et al. 2008), nearly two-thirds of all alternative exons are conserved up to the common ancestor of C. elegans and C. japonica (Fig. 5B). However, conservation of both classes of alternative exon triplets dropped substantially to $\sim 30 \%$ in alignments between the $C$. elegans and C. angaria genomes (Fig. 5B). Tissue-regulated alternative exons and their neighboring exons were found to be conserved at a modest, but consistently higher rate throughout the phylogeny compared with non-tissue-regulated alternative exons (Fig. 5B). In particular, C. japonica has a significantly greater proportion of conserved tissue-regulated exon triplets with C. elegans compared with non-tissue-regulated alternative splicing events $(72 \%$ vs. $59 \%$, respectively; $P$-value $=$ 0.02, Fisher's exact test) (Fig. 5B).

Alternative splicing is known to be regulated by cis elements in exonic and intronic regions. As such, selection pressure to preserve these elements would increase conservation in the intronic regions surrounding alternative exons compared with analogous regions flanking constitutive exons. We investigated this characteristic by measuring average conservation in introns on an individual nucleotide level across the seven species described above using the program phyloP (Pollard et al. 2010). We focused on the $23 \mathrm{nt}$ adjacent to each splice site, because this length would still partition our smallest introns ( $40 \mathrm{nt}$ in length) into $5^{\prime}$ and $3^{\prime}$ halves. By comparing phyloP scores of $23 \mathrm{nt}$ adjacent to each

other group consisted of non-tissue-regulated alternative exons and their flanking sequences, for which our TRAP-seq measurements suggest the alternative exons show little or no tissue-specific splicing differences. Collectively, our analysis included 113 tissue-regulated exons, 277 non-tissue-regulated exons, and 467 constitutive exons for comparison (for details, see Methods) (Fig. 5A).

Multiple sequence alignments of C. elegans exon triplets were performed with syntenic sequences from six species in the Caenorhabditis genus: Caenorhabditis briggsae, Caenorhabditis remanei, Caenorhabditis tropicalis, Caenorhabditis brenneri, Caenorhabditis japonica, and Caenorhabditis angaria (Fig. 5A). First, we investigated the degree of conservation of exon triplets from each group of splicing events, defined by the presence of all three exons and alignment of the splice sites surrounding these exons. As expected, we see a decrease in the number of conserved exon triplets as evolutionary divergence from C. elegans increases (Fig. 5A). We next calculated the percentage of conservation of exon triplets between C. elegans and the other species, excluding genes that had no clear splice site, we observed increased sequence conservation surrounding alternative exons compared with constitutive exons (Fig. 5C,D). This increased conservation was particularly apparent in the splice sites and neighboring regions immediately adjacent to the alternative exons (comparing constitutive exons with both classes of alternative exons, at $3^{\prime}$ end of upstream intron or $5^{\prime}$ end of downstream intron; $P$-values $<2.2 \times 10^{-16}$, Wilcoxon signed-rank test) (Fig. 5C,D). Moreover, the intronic regions immediately flanking tissue-regulated alternative exons were significantly more conserved than the corresponding sequences flanking non-tissue-regulated exons ( 3 ' end of upstream intron, $P$-value $<2 \times 10^{-6} ; 5^{\prime}$ end of downstream intron, $P$-value $<2.2 \times$ $10^{-16}$; Wilcoxon signed-rank test) (Fig. 5C,D).

Taken together, these data suggest that the intronic regions surrounding alternative exons are considerably more conserved than analogous sequences in constitutive exons. Moreover, the extended conservation required for regulation of tissue-specific exons likely creates selection pressure to preserve neighboring exon-intron architecture across evolutionary timescales. 
Alternative and tissue-regulated exons are enriched in motifs recognized by RBPs

Previous studies in C. elegans have shown that sequence enrichment search strategies can be effective at identifying candidate splicing regulatory signals (Kabat et al. 2006; Ramani et al. 2011). We used hypergeometric optimization of motif enrichment (HOMER) (Heinz et al. 2010), which can detect enriched motifs in a given list of sequences relative to a background list of sequences. We first compared sequences spanning alternative cassette exons and flanking introns to equivalent sequences surrounding constitutively included exons (Fig. 6A). The resulting enriched sequences yield several expected motifs previously identified (Kabat et al. 2006; Ramani et al. 2011) and recognized by characterized RBPs, including FOX-1/Rbfox and the muscle-specific regulator SUP-12 (an ortholog of Rbm24 and Rbm38), which have both been implicated in alternative splicing regulation in C. elegans (Kuroyanagi et al. 2007). We also identified enriched cis elements recognized by UNC-75/CELF and EXC-7 (an ortholog of Elavl4/HuD), both known to regulate tissue-specific alternative splicing within the nervous system (Kuroyanagi et al. 2013a,b; Norris et al. 2014; Chen et al. 2016).

We next searched for motifs enriched in tissue-regulated alternative splicing events relative to analogous sequences spanning non-tissue-regulated splicing events (Fig. 6B). Again, we identified motifs for known RBPs and splicing regulators. Some of these motifs were not identified in our alternative versus constitutive exon comparison above, suggesting that they are selectively associated with tissue-regulated splicing patterns.

In both comparisons, we also found enrichment of many motifs that could not be connected with a cognate RBP, as well as motifs that matched consensus sequences from other species (Fig. 6A,B). Taken together, our results indicate that alternative exons and, by extension, tissue-regulated exons, are coordinately regulated by RBPs, several of which remain to be discovered and characterized.

\section{UNC-75/CELF recognizes conserved motifs overlapping a $5^{\prime}$ splice site and represses exon inclusion}

Both our conservation analysis (Fig. 5) and motif enrichment analysis (Fig. 6) indicated that tissue-regulated exons are flanked by conserved and overrepresented sequence features, likely reflecting the presence of critical cis elements. As such, we next assessed whether these analyses could prove useful in uncovering novel splicing regulatory mechanisms.
We identified conserved UNC-75 consensus sequences overlapping the $5^{\prime}$ splice site in the intron downstream from alternative exon 9 of the zoo-1 gene (Fig. 7; Supplemental Fig. S5). This gene encodes an ortholog of the zonula occludens tight junctional protein ZO-2, a key protein involved in cell adhesion and cell proliferation (González-Mariscal et al. 2019). Our TRAP-seq data identified that zoo- 1 exon 9 is primarily excluded in neurons and mostly included in muscle cells (Fig. 7A). We further validated these patterns by coexpressing neuron and muscle promoter driven zoo-1 two-color splicing reporters (Fig. 7A). Consistent with our TRAP-seq measurements, when signal from both cell types are imaged together, we detected significantly preferential inclusion of exon 9 (mCherry) in muscle cells and more biased skipping of exon 9 (GFP) in neuronal cells (Fig. 7B).
A

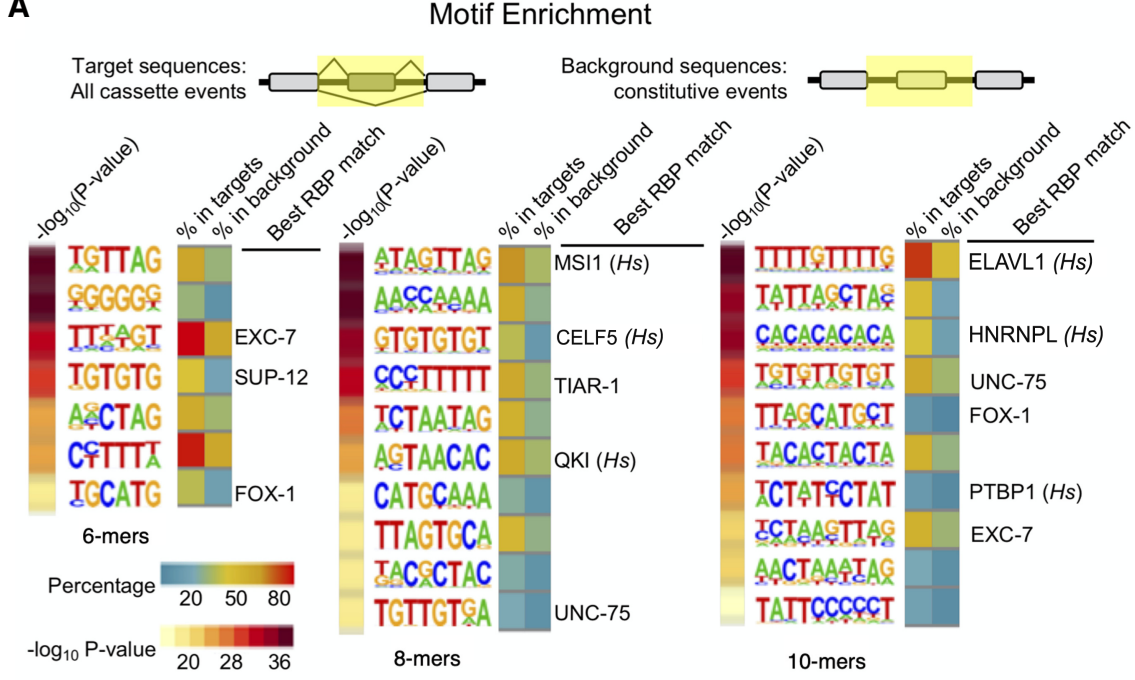

\section{B}

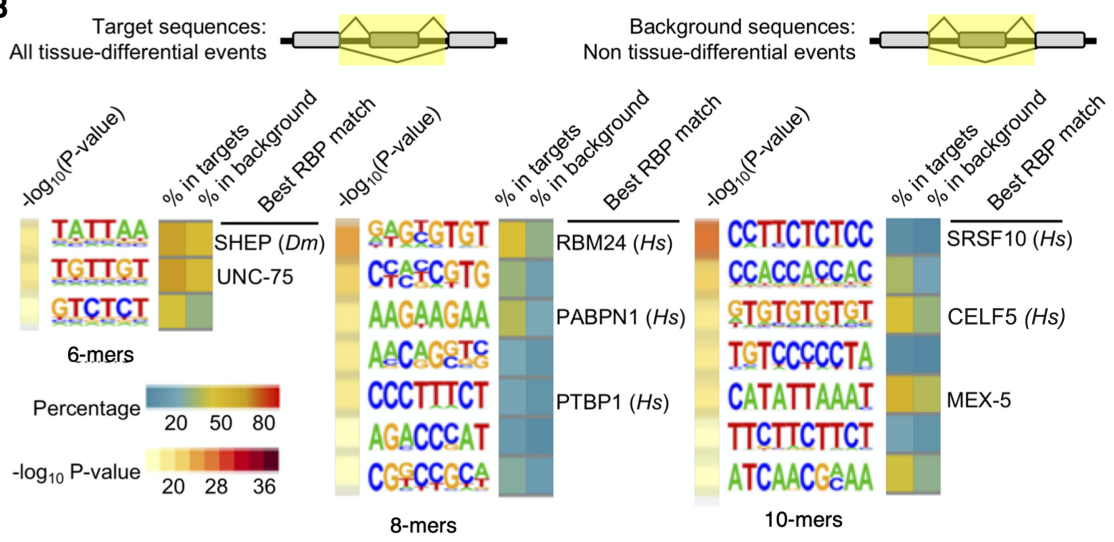

Figure 6. Enriched cis elements surrounding alternative exons and tissue-regulated alternative exons. (A) Enriched motifs identified when comparing all cassette-type alternative exons with constitutive exons. Top panel displays the regions compared (highlighted in yellow) spanning internal exon and flanking introns. Enrichment $P$-values are listed to the left of the motifs as $-\log _{10}$-transformed values (yellowred scale). Motifs are presented as sequence logos derived from cis elements observed in the data. The percentage of sequences in the target group versus the background group are displayed to the right of the motif logos (blue-yellow-red scale). If a sequence motif overlaps with a known consensus motif for an RBP identified, the RBP is labeled as well as the species the consensus was derived from. $(\mathrm{Hs})$ Homo sapiens. (B) Same as in $A$, except target sequences are tissue-regulated alternative splicing events and background sequences are non-tissue-regulated alternative splicing events. $(\mathrm{Hs})$ Homo sapiens, $(\mathrm{Dm})$ D. melanogaster. 
Given that UNC-75 is broadly expressed in the nervous system (Loria et al. 2003), we speculated that it has a direct role in blocking exon 9 inclusion through interaction with its cognate cis elements. To test this hypothesis, we expressed a zoo-1 exon 9 splicing reporter in the nervous system of wild-type animals, animals lacking unc-75, or animals lacking asd-1, an unrelated RBP that is also expressed in the nervous system. RT-PCR assays amplifying both zoo-1 isoforms were then performed on total RNA collected from these animals (Fig. 7C). Consistent with our prediction, exon 9 is more highly included in the neurons of unc-75 loss-of-function mutants compared with wild-type animals

A
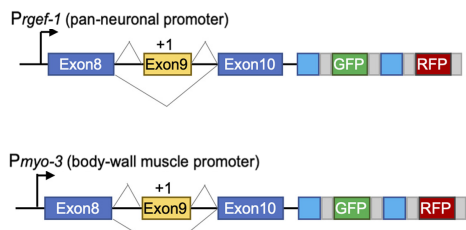

C

zoo-1 splicing reporter expressed in neurons

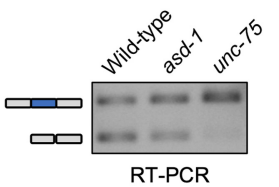

D

UNC-75 consensus

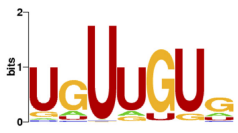

RNAcompete

F

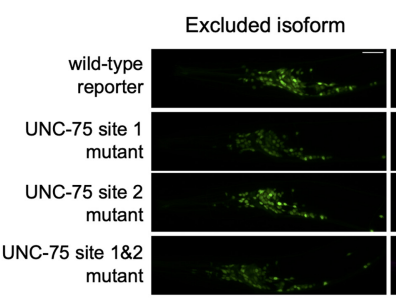

zoo-1 splicing reporters expressed in neurons ...GAGAUCCGAGGUGUUGUGCCGUGCUUUGA... UNC-75 site 2 mutant ...GAGAUCCGAGGUGCCGUGCCGUGCUUUGA... UNC-75 site $1 \& 2$ mutant

E
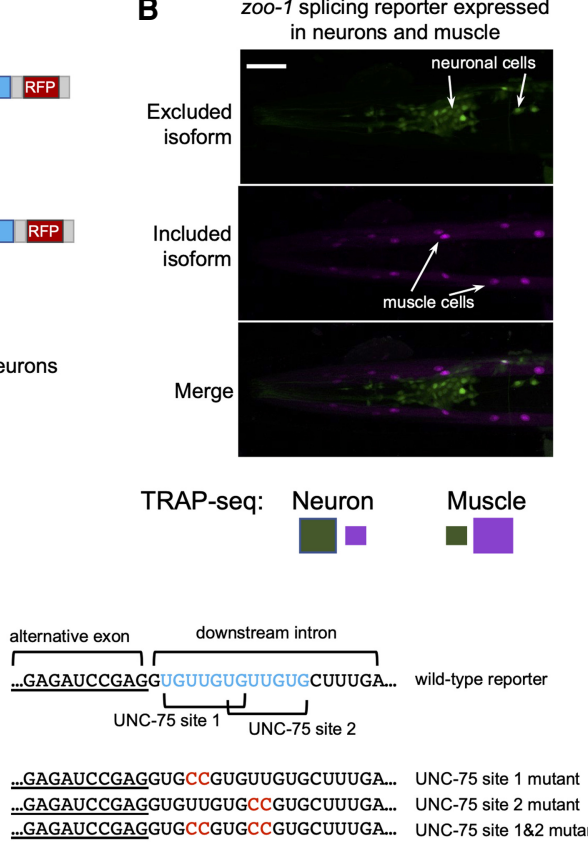

wild-type animals, suggesting that loss of unc-75 has a specific ef-

fect on zoo-1 exon 9 alternative splicing (Fig. 7C). We next generated two-color splicing reporters with mutations targeting one or both UNC-75 consensus sequences (Fig. 7D,E) and drove expression of these reporters specifically in neurons (Fig. 7F). When imaging the neuron-expressed reporters in the absence of any muscle signal, the included isoform can now be detected with imaging settings that would otherwise saturate signal in muscle cells. We next quantified the total fluorescence signal through the head regions of animals expressing wild-type and mutant reporters (Fig. 7F,G). These experiments showed that mutations in the first or second cis elements increased the relative proportion of the included isoform in neurons (Fig. 7F,G). However, mutations targeting both cis elements led to the maximum effect on splicing, in which exon 9 is significantly more included in most neurons $(P$-value $<0.01$, Kruskal-Wallis rank sum test) (Fig. 7F,G).

Taken together, these results indicate that CELF proteins can act as repressors of alternative exon inclusion in the nervous system when bound to cis elements directly overlapping with $5^{\prime}$ splice sites.

\section{Alternative exons and tissue-biased exons have distinct regulatory features compared with constitutive exons}

Previous studies of vertebrate alternatively spliced exons and their flanking introns and exons have indicated that regulated exons have distinct sequence characteristics compared with analogous regions surrounding constitutively spliced exons (Xing and Lee 2005; Yeo et al. 2005; Yura et al. 2006; Keren et al. 2010; Barbosa-Morais et al. 2012). In a similar manner, we compared various sequence features among alternative exons, constitutive exons, and their surrounding sequences.

First, we tested the frequency of internal constitutive or alternative exons to preserve the open reading framespecifically, if the exon is a multiple of 3 nt (Fig. 8A). Tissue-regulated alternative exons have been shown to have increased selection pressure to be framepreserving in order to be included or skipped in transcripts without causing a shift in reading frame (Modrek et al. 2001). Consistent with these observations, we observe an increase in the frequency of alternative exons to preserve reading frame relative to constitutive exons (Fig. 8A). Frame preservation frequency is further increased among the 
A
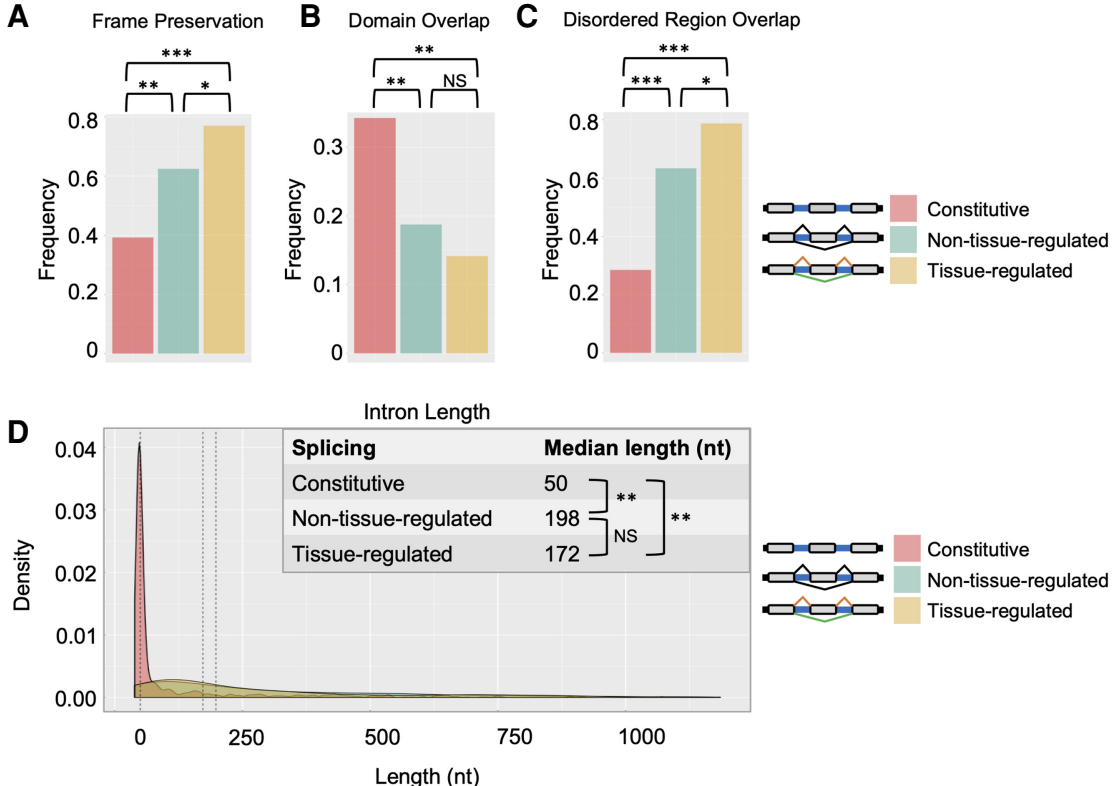
वित्रि Tissue-regulated

$\mathbf{E}$

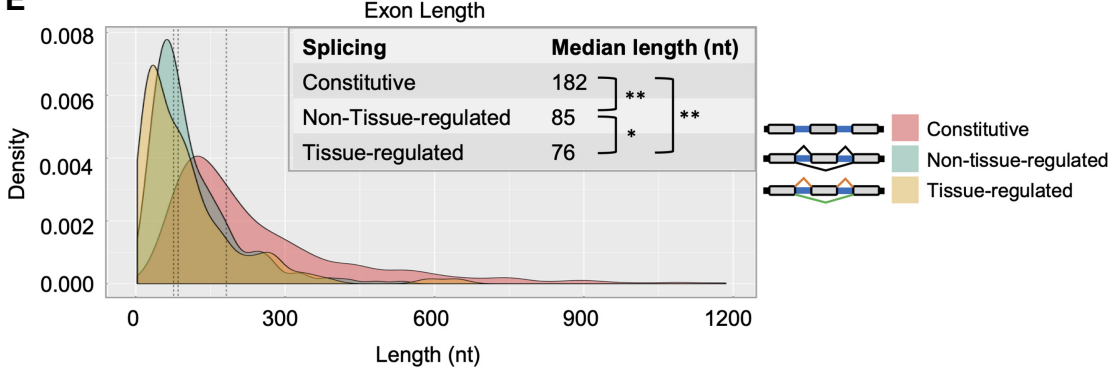

Figure 8. Features associated with alternative exons and tissue-regulated alternative exons. For panels $A-E$, comparisons were made between constitutively spliced exons (pink), alternative exons (light blue), and tissue-regulated alternative exons (gold). ( $A$ ) Comparison of frequency of frame preservation (percentage of exons that are a multiple of $3 \mathrm{nt}$ ). (B) Frequency of exons overlapping with a UniProt conserved protein domains. (C) The frequency of exons overlapping with predicted disordered regions in proteins. $(D)$ Distributions of the lengths of introns flanking internal exons from same comparison groups. (E) Distribution of the lengths of exons. $(A, C)$ Pearson's chi-squared test: $\left(^{*}\right) P \leq 0.01 ;\left(^{* *}\right) P<1$ $\times 10^{-3} ;\left({ }^{* * *}\right) P<1 \times 10^{-10}$. $(D, E)$ Wilcoxon rank-sum test: $\left(^{*}\right) P \leq 0.05 ;\left(^{* *}\right) P<1 \times 10^{-15}$.

tissue-regulated splicing events, in which $\sim 80 \%$ of exons are multiples of three.

We next tested the frequency with which internal constitutive or alternative exons overlap with conserved protein domains or intrinsically disordered regions (IDRs) (Fig. 8B,C). We found that alternative exons overlap less frequently with annotated protein domains (Fig. 8B) but more frequently overlap with IDRs, with tissue-regulated alternative exons having the highest degree of overlap with IDRs (approaching 80\% of all exons) (Fig. 8C). Again, this trend is consistent with reports in vertebrates, where alternatively spliced exons are known to modulate protein-protein interactions and the formation of multivalent protein assemblies through altering IDR sequence features (Romero et al. 2006; Buljan et al. 2012; Ellis et al. 2012; Yang et al. 2016).

Finally, we measured the lengths of internal constitutive exons, alternative exons, and their flanking intronic regions (Fig. 8D,E). Consistent with previous observations (Fox-Walsh et al. 2005; Kabat et al. 2006; Kim et al. 2007), introns flanking both tissue-differential and non-tissue-differential alternative exons are significantly longer than introns flanking constitutively spliced
Constitutive Non-tissue-regulated

exons (Fig. 8D). This tendency toward longer intron length is preserved in both upstream and downstream introns flanking alternative exons, with the downstream intron having a somewhat longer length (Supplemental Fig. S6). In contrast, we found that alternative exons are significantly smaller in length compared with constitutive exons (Fig. 8E). This tendency toward a shorter length was also more pronounced in the tissuedifferential alternative exons.

Collectively, these results show that alternative exons and their flanking intronic sequences have features that distinguish them from constitutive exons. Moreover, these identified features show hallmarks shared by other invertebrate and vertebrate alternative exons, suggesting common evolutionary constraints and functional consequences of these exons across metazoans.

Microexons are differentially regulated across cell types and highly conserved across Caenorhabditis species

Our observations above indicated that tissue-regulated exons tend to be shorter than other classes of exons. Upon closer examination, we identified 24 microexons ( $\leq 27 \mathrm{nt}$ in length) among these tissue-regulated exons (Fig. 9; Supplemental Table S7). Microexons have recently been identified as an interesting class of exons, with distinct mechanisms controlling their splicing patterns, and have shown roles in neuronal development and physiology in vertebrates (Scheckel and Darnell 2015). Moreover, the aberrant regulation of these exons has been associated with autism spectrum disorders (Irimia et al. 2014; Quesnel-Vallières et al. 2016; Gonatopoulos-Pournatzis et al. 2018).

Our identification of tissue-regulated microexons suggested that there may be additional microexons in our transcriptome data. We therefore slightly relaxed our filtering criteria and detected an additional 43 alternatively spliced microexons, for a total of 67 (Supplemental Table S7), indicating that this class of exons may be difficult to comprehensively detect in typical transcriptome analysis with short read data from whole-animal samples. Roughly one-third (23/67) microexons were detected exclusively in our tissue-enriched TRAP-seq data, suggesting that microexons often undergo tissue-differential splicing.

We next assessed whether microexons possessed features that distinguished them from larger alternative exons ( $\geq 99 \mathrm{nt})$. Microexons exhibit a high frequency of reading frame preservation (>80\%) compared with larger exons (Fig. 9A). Additionally, an examination of intronic regions revealed that sequences surrounding microexons, particularly the $3^{\prime}$ end of the upstream intron and $5^{\prime}$ end of the downstream intron, are considerably more conserved than corresponding regions surrounding larger 
A

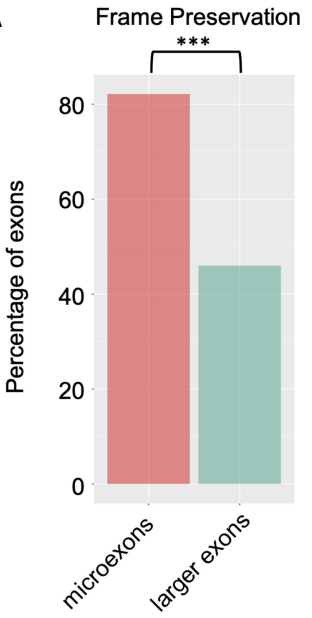

B

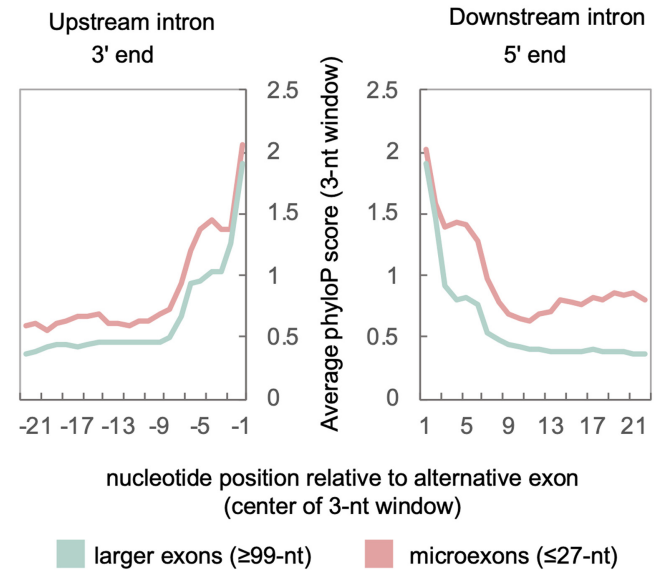

C

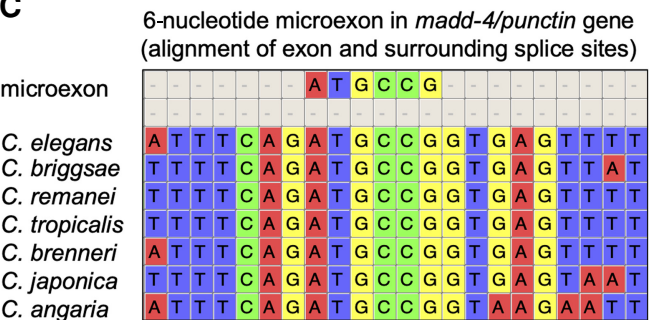

D

15-nucleotide microexon in spc-1/spectrin gene (alignment of exon and surrounding splice sites)

microexon - ACTAACATGGCAGAA

C. elegans

C. briggsae

C. remanei

C. tropicalis

C. brenneri

C. japonica

C. angaria

Figure 9. Microexons $<27 \mathrm{nt}$ are highly conserved in Caenorhabditis species. (A) Comparison of frame preservation frequency among microexons (pink) and larger exons (green). $\left({ }^{* * *}\right) P<1 \times 10^{-10}$, Pearson's chi-squared test. (B) Plot of average phyloP score (rolling 3-nt average) over last $23 \mathrm{nt}$ (left panel) and first $23 \mathrm{nt}$ (right panel) of the upstream and downstream introns flanking microexons (pink) or larger exons (green), respectively. (C) A multiple sequence alignment displaying a highly conserved 6-nt microexon and its surrounding splice sites in the madd-4 gene. $(D)$ A multiple sequence alignment of a highly conserved 15-nt microexon and its surrounding splice sites in the spc-1 gene.

alternative exons ( $P$-values all $<1 \times 10^{-7}$, Wilcoxon signed-rank test) (Fig. 9B). Representative multiple sequence alignments provide another view of this conservation. For example, the 6-nt microexon in the madd-4/punctin gene and the 15-nt microexon in the $s p c$-1 gene (a spectrin ortholog) were both found to show high levels of conservation within the exon and flanking intronic regions among all Caenorhabditis species examined (Fig. 9C,D).

Taken together, our analysis has shed light on an important class of exons that are likely influencing aspects of protein function and diversity.

\section{Discussion}

In this study, we have highlighted the utility of the TRAP-seq approach for transcriptome-wide studies of features associated with

alternative splicing across tissues and specific neuronal cell types. Our samples are also sequenced to fairly high depths of coverage (Supplemental Fig. S1), which has facilitated our ability to detect splice junctions with increased confidence. Consistent with this point, we downsampled our short read data and showed that, although we are approaching saturation in our ability to detect alternatively spliced junctions (Supplemental Fig. S7), our current results are likely underestimating the full complexity of splicing in the isolated tissues of the organism. Our method provides a robust and complementary avenue for obtaining mRNA profiles along with other approaches such as mRNA tagging (Spencer et al. 2011; Blazie et al. 2015) and fluorescence-activated cell sorting of labeled cells (Spencer et al. 2014; Kaletsky et al. 2016).

Our results show that cells of the nervous system had the highest numbers of detected splice junctions (Fig. 1C), mirroring findings in vertebrates indicating that the nervous system exhibits relatively high levels of alternative splicing compared with other tissues (BarbosaMorais et al. 2012; Tapial et al. 2017). It is only recently that neuronal-subtype differences in splicing have been interrogated and have revealed differences in isoform expression (Wamsley et al. 2018; Wang et al. 2018; Furlanis et al. 2019; Saito et al. 2019). Taken together, these observations and our own results suggest that an expanded use of alternative splicing is a hallmark of metazoan nervous systems, including the relatively compact nervous system of $C$. elegans.

Our observation that tissue-regulated splicing is enriched in complex events (Fig. 3A,B) in C. elegans is consistent with a recent study indicating that complex events account for a significant proportion of conserved differential splicing variation across mammalian tissues (Vaquero-Garcia et al. 2016). It is interesting to speculate how complex splicing events may arise during evolution. One likely model would involve complex events emerging from simpler ancestral splicing events. Comparisons of genomic and transcript sequences across multiple species may be able to provide some support of this model.

We identified highly conserved UNC-75/CELF binding sites overlapping directly with the $5^{\prime}$ splice site flanking alternative exon 9 of the zoo- 1 gene. We speculate a mechanism in which UNC-75 binds to these cis elements to repress inclusion of the alternative exon in neurons (Fig. 7). This mechanism of repression contrasts with the role of UNC-75 as an activator of exon inclusion when bound to a cis element 30 bases downstream from the $5^{\prime}$ splice site in the unc-16 gene (Norris et al. 2014). Recent studies have revealed position-dependent regulation of splicing by

\section{Genome Research}

www.genome.org 
UNC-75 (Kuroyanagi et al. 2013b). Thus, small differences in the location and position of the UNC-75 cis elements can have distinct consequences on splicing regulation even within the same intronic region. It will be interesting to explore the biochemical mechanisms of UNC-75 binding to target mRNAs and investigate how prevalent this mode of CELF-mediated splicing repression is in multicellular animals.

Our results suggest that there are shared constraints across protein coding genes in multicellular animals that lead to the evolution of regulated splicing patterns. In particular, the possibility that tissue-regulated exons are more likely to be selected for influencing disordered regions in proteins is intriguing. Several emerging roles for disordered protein sequences include shaping protein-protein interactions and biophysical properties, as well as serving as sites of post-translational modifications (Wright and Dyson 2015). Given that tissue-regulated exons are highly conserved, their influence on protein function is likely important. An important goal will be to determine the molecular consequences of these splicing events on protein function in vivo.

Microexons have emerged as important modulators of protein function in vertebrates, particularly in the nervous system (Irimia et al. 2014; Li et al. 2015). Our current analysis has expanded the number of known microexons in C. elegans. Moreover, given that these microexons are highly conserved and often framepreserving (Fig. 9), it is highly likely that these exons will influence protein function in different tissues, as recent studies in vertebrates have confirmed (Parras et al. 2018; Gonatopoulos-Pournatzis et al. 2020). It was proposed in a recent study that the explosion of microexons included in neuronal transcripts may have coincided with the evolution of the enhancer of microexons (eMIC) domain in the serine/arginine repetitive matrix (SRRM) protein family (Torres-Méndez et al. 2019). Other studies have also identified additional factors controlling the splicing of these exons ( $\mathrm{Li}$ et al. 2015; Gonatopoulos-Pournatzis et al. 2018). However, SRRM proteins in Caenorhabditis species lack the eMIC domain (TorresMéndez et al. 2019). Thus, C. elegans represents an interesting model system to explore ancient mechanisms governing microexon splicing.

Collectively, our work has revealed that alternative splicing represents a rich layer of gene expression, contributing to the specialized functions of cell and tissue types in C. elegans, particularly in the nervous system. As isoform-sensitive transcriptome profiling approaches at single-cell resolution are starting to emerge, we will likely gain an even deeper appreciation for the extent to which transcript diversity will ultimately impact the proteome and cellular specialization.

\section{Methods}

\section{C. elegans maintenance and strains used in this study}

Animals were maintained at $21^{\circ} \mathrm{C}$ and grown on nematode growth media plates seeded with OP50-1 bacteria under standard conditions (Brenner 1974). In addition to the N2 wild-type strain, the list of strains used in the current study is listed in Supplemental Table S8.

\section{TRAP-seq and read alignment}

cDNA libraries from whole-animal input lysates and tissue-enriched data sets were created as described previously (Gracida et al. 2017). FASTQ files from paired-end Illumina sequencing data were used as input files, and reads were mapped using STAR aligner (Dobin et al. 2013), an algorithm capable of high-speed read alignment and splice junction detection. Genome index files were generated using C. elegans genome annotation WS251 and the default parameters. Two-pass read alignment was performed to increase detection of novel splice junctions. SAM and sorted BAM files were generated for subsequent analysis. For additional analysis to identify tissue- and neuron-subtype unique junction reads, see the Supplemental Material.

Predicting tissue-differential splicing, categorizing splicing events into splicing classes, and characterization of splicing events

By using sorted BAM and BAM index files from our aligned reads, the Majiq/Voila software pipeline (Vaquero-Garcia et al. 2016) was applied to our TRAP-seq data to identify and visualize alternative splicing events (reported as local splicing variations [LSVs], within a single tissue) and differential splicing events (between two or more tissues). By using the output files of the Majiq analysis, more stringent filters were applied in order to obtain a set of high-confidence alternative splicing and differential splicing events for further computational analysis. All coding, statistical tests, and graphing were performed using R 3.5.1 (R Core Team 2018). For additional details, see Supplemental Material.

\section{Motif enrichment analysis}

Motif analysis was performed using HOMER (Heinz et al. 2010) to discover annotated or de novo motifs that were enriched in tissueregulated splicing events, using sets of constitutive splicing events or non-tissue-regulated splicing events as background sets. For specific details, see Supplemental Material.

\section{GO overrepresentation analysis}

We used the online tool, WebGestalt (Liao et al. 2019) to perform the overrepresentation analyses. Sample data sets were (1) genes that are differentially spliced between any two tissues $(\Delta \mathrm{PSI} \geq$ 0.15 ), and (2) genes that are differentially expressed between any two tissues (with a $5 \times$ up- or down-regulated). In all comparisons, we search for enrichment against background gene sets in which genes were filtered for expression (above 50 read counts) within the tissue(s) involved. This filter controlled for simply enriching for genes that were differentially expressed in particular tissues. Overrepresentation analysis was performed to identify significantly enriched biological processes (Bonferroni multiple testing correction, corrected $P$-values $<0.05)$. This analysis was performed for differentially spliced/differentially expressed genes among the broad tissues and within the nervous system.

\section{Construction of two-color splicing reporter plasmids, microinjection, microscopy, and quantification}

Our two-color reporters were generated using standard molecular biology approaches, integrated into animals, and visualized and quantified by confocal microscopy. For a list of primers used in the study, see Supplemental Table S9. For a list of plasmids used, see Supplemental Table S8. For additional details, please see Supplemental Material and Supplemental Figure S8.

\section{Multiple sequence alignments and analysis of sequence conservation}

For multiple sequence alignments, we obtained coordinates of all relevant splicing events from our TRAP-seq data (spanning three exons and two introns, in which the internal exon is either an alternative or constitutive exon). By using these coordinates, corresponding BED files were generated and used to obtain multiple 
alignment format (MAF) files from the 26-way nematode alignment track on genome assembly ce11 (WBCel235) from the University of California Santa Cruz (UCSC) Genome Browser. We then extracted homologous sequences from seven species (C. elegans, C. brenneri, C. briggsae, C. remanei, C. japonica, C. tropicalis, and C. angaria) and realigned relevant regions surrounding alternative or constitutive exons using MUSCLE (Edgar 2004). To measure conservation patterns in a base-by-base manner, we used the program phyloP (Pollard et al. 2010), a part of the "rphast" R package (Hubisz et al. 2011). For additional details, see Supplemental Material.

\section{Data access}

All raw and processed sequencing data generated in this study have been submitted to the NCBI Gene Expression Omnibus (GEO; https://www.ncbi.nlm.nih.gov/geo/) under accession number GSE106374.

\section{Competing interest statement}

The authors declare no competing interests.

\section{Acknowledgments}

We thank John Laver and Arneet Saltzman for critical reading of the manuscript. B.K. is supported by a Natural Sciences and Engineering Research Council of Canada (NSERC) PGS Doctoral fellowship. Research in the Calarco Laboratory is also generously supported by the Canadian Foundation for Innovation, the Ontario Research Fund, the Canada First Research Excellence Fund (Medicine by Design), and the Canada Research Chairs Program. Some of the analysis described in this study was performed on high-performance computing hardware in the Moses Research Laboratory (University of Toronto) supported by the Canada Foundation for Innovation. This work was supported by funding by an NSERC discovery grant (2017-06573) and a Canadian Institutes of Health Research (CIHR) Project grant (PJT156300), awarded to J.A.C. This research was enabled in part by support provided by Compute Ontario (https://computeontario .ca/) and Compute Canada (www.computecanada.ca).

Author contributions: B.K., P.P.P., X.G., and J.A.C. conceived of the study and conducted experiments. B.K., P.P.P., and J.A.C. performed data analysis, assembled figures, and wrote the manuscript with input from X.G. and Y.Z. L-M.S. helped with initial construction of two-color splicing reporters. I.P. helped with analysis of overlap of alternative exons with regions encoding intrinsically disordered proteins.

\section{References}

Ahier A, Jarriault S. 2014. Simultaneous expression of multiple proteins under a single promoter in Caenorhabditis elegans via a versatile 2A-based toolkit. Genetics 196: 605-613. doi:10.1534/genetics.113.160846

Baeza-Centurion P, Miñana B, Schmiedel JM, Valcárcel J, Lehner B. 2019. Combinatorial genetics reveals a scaling law for the effects of mutations on splicing. Cell 176: 549-563.e23. doi:10.1016/j.cell.2018.12.010

Barash Y, Calarco JA, Gao W, Pan Q, Wang X, Shai O, Blencowe BJ, Frey BJ. 2010. Deciphering the splicing code. Nature 465: 53-59. doi:10.1038/ nature09000

Barberan-Soler S, Zahler AM. 2008. Alternative splicing regulation during $C$. elegans development: splicing factors as regulated targets. PLoS Genet 4 e1000001. doi:10.1371/journal.pgen.1000001

Barberan-Soler S, Lambert NJ, Zahler AM. 2009. Global analysis of alternative splicing uncovers developmental regulation of nonsense-mediated decay in C. elegans. RNA 15: 1652-1660. doi:10.1261/rna.1711109
Barbosa-Morais NL, Irimia M, Pan Q, Xiong HY, Gueroussov S, Lee LJ, Slobodeniuc V, Kutter C, Watt S, Çolak R, et al. 2012. The evolutionary landscape of alternative splicing in vertebrate species. Science 338: 1587-1593. doi:10.1126/science.1230612

Blazie SM, Babb C, Wilky H, Rawls A, Park JG, Mangone M. 2015. Comparative RNA-Seq analysis reveals pervasive tissue-specific alternative polyadenylation in Caenorhabditis elegans intestine and muscles. BMC Biol 13: 4. doi:10.1186/s12915-015-0116-6

Blazie SM, Geissel HC, Wilky H, Joshi R, Newbern J, Mangone M. 2017. Alternative polyadenylation directs tissue-specific miRNA targeting in Caenorhabditis elegans somatic tissues. Genetics 206: 757-774. doi:10 .1534/genetics.116.196774

Brenner S. 1974. The genetics of Caenorhabditis elegans. Genetics 77: 71-94.

Brown JB, Boley N, Eisman R, May GE, Stoiber MH, Duff MO, Booth BW, Wen J, Park S, Suzuki AM, et al. 2014. Diversity and dynamics of the Drosophila transcriptome. Nature 512: 393-399. doi:10.1038/ nature 12962

Buljan M, Chalancon G, Eustermann S, Wagner GP, Fuxreiter M, Bateman A, Babu MM. 2012. Tissue-specific splicing of disordered segments that embed binding motifs rewires protein interaction networks. Mol Cell 46: 871-883. doi:10.1016/j.molcel.2012.05.039

Chen L, Liu Z, Zhou B, Wei C, Zhou Y, Rosenfeld MG, Fu XD, Chisholm AD, Jin Y. 2016. CELF RNA binding proteins promote axon regeneration in C. elegans and mammals through alternative splicing of syntaxins. eLife 5: e16072. doi:10.7554/eLife.16072

Dobin A, Davis CA, Schlesinger F, Drenkow J, Zaleski C, Jha S, Batut P, Chaisson M, Gingeras TR. 2013. STAR: ultrafast universal RNA-seq aligner. Bioinformatics 29: 15-21. doi:10.1093/bioinformatics/bts635

Edgar RC. 2004. MUSCLE: multiple sequence alignment with high accuracy and high throughput. Nucleic Acids Res 32: 1792-1797. doi:10.1093/ nar/gkh340

Ellis JD, Barrios-Rodiles M, Çolak R, Irimia M, Kim TH, Calarco JA, Wang X, Pan Q, O"Hanlon D, Kim PM, et al. 2012. Tissue-specific alternative splicing remodels protein-protein interaction networks. Mol Cell 46: 884-892. doi:10.1016/j.molcel.2012.05.037

Fagnani M, Barash Y, Ip JY, Misquitta C, Pan Q, Saltzman AL, Shai O, Lee L, Rozenhek A, Mohammad N, et al. 2007. Functional coordination of alternative splicing in the mammalian central nervous system. Genome Biol 8: R108. doi:10.1186/gb-2007-8-6-r108

Fox-Walsh KL, Dou Y, Lam BJ, Hung SP, Baldi PF, Hertel KJ. 2005. The architecture of pre-mRNAs affects mechanisms of splice-site pairing. Proc Natl Acad Sci 102: 16176-16181. doi:10.1073/pnas.0508489102

Fu XD, Ares M. 2014. Context-dependent control of alternative splicing by RNA-binding proteins. Nat Rev Genet 15: 689-701. doi:10.1038/ $\operatorname{nrg} 3778$

Furlanis E, Traunmüller L, Fucile G, Scheiffele P. 2019. Landscape of ribosome-engaged transcript isoforms reveals extensive neuronal-cellclass-specific alternative splicing programs. Nat Neurosci 22: 17091717. doi:10.1038/s41593-019-0465-5

Gerstberger S, Hafner M, Tuschl T. 2014. A census of human RNA-binding proteins. Nat Rev Genet 15: 829-845. doi:10.1038/nrg3813

Gerstein MB, Lu ZJ, Van Nostrand EL, Cheng C, Arshinoff BI, Liu T, Yip KY, Robilotto R, Rechtsteiner A, Ikegami K, et al. 2010. Integrative analysis of the Caenorhabditis elegans genome by the modENCODE project. Science 330: $1775-1787$. doi:10.1126/science.1196914

Giudice J, Xia Z, Wang ET, Scavuzzo MA, Ward AJ, Kalsotra A, Wang W, Wehrens XHT, Burge CB, Li W, et al. 2014. Alternative splicing regulates vesicular trafficking genes in cardiomyocytes during postnatal heart development. Nat Commun 5: 3603. doi:10.1038/ncomms4603

Gonatopoulos-Pournatzis T, Wu M, Braunschweig U, Roth J, Han H, Best AJ, Raj B, Aregger M, O'Hanlon D, Ellis JD, et al. 2018. Genome-wide CRISPR-Cas9 interrogation of splicing networks reveals a mechanism for recognition of autism-misregulated neuronal microexons. Mol Cell 72: 510-524.e12. doi:10.1016/j.molcel.2018.10.008

Gonatopoulos-Pournatzis T, Niibori R, Salter EW, Weatheritt RJ, Tsang B, Farhangmehr S, Liang X, Braunschweig U, Roth J, Zhang S, et al. 2020. Autism-misregulated eIF4G microexons control synaptic translation and higher order cognitive functions. Mol Cell 77: 1176-1192.e16. doi:10.1016/j.molcel.2020.01.006

González-Mariscal L, Gallego-Gutiérrez H, González-González L, Hernández-Guzmán C. 2019. ZO-2 is a master regulator of gene expression, cell proliferation, cytoarchitecture, and cell size. Int J Mol Sci 20: 4128. doi:10.3390/ijms20174128

Gracida X, Calarco JA. 2017. Cell type-specific transcriptome profiling in $C$. elegans using the translating ribosome affinity purification technique. Methods 126: 130-137. doi:10.1016/j.ymeth.2017.06.023

Gracida X, Norris AD, Calarco JA. 2016. Regulation of tissue-specific alternative splicing: C. elegans as a model system. Adv Exp Med Biol 907: 229261. doi:10.1007/978-3-319-29073-7_10

\section{Genome Research}

www.genome.org 
Gracida X, Dion MF, Harris G, Zhang Y, Calarco JA. 2017. An ElonginCullin-SOCS Box complex regulates stress-induced serotonergic neuromodulation. Cell Rep 21: 3089-3101. doi:10.1016/j.celrep.2017.11.042

Heiman M, Schaefer A, Gong S, Peterson JD, Day M, Ramsey KE, SuárezFariñas M, Schwarz C, Stephan DA, Surmeier DJ, et al. 2008. A translational profiling approach for the molecular characterization of CNS cell types. Cell 135: 738-748. doi:10.1016/j.cell.2008.10.028

Heinz S, Benner C, Spann N, Bertolino E, Lin YC, Laslo P, Cheng JX, Murre C, Singh H, Glass CK. 2010. Simple combinations of lineage-determining transcription factors prime cis-regulatory elements required for macrophage and B cell identities. Mol Cell 38: 576-589. doi:10.1016/j .molcel.2010.05.004

Hobert O. 2010. Neurogenesis in the nematode Caenorhabditis elegans. WormBook 1-24. doi:10.1895/wormbook.1.12.2

Hubisz MJ, Pollard KS, Siepel A. 2011. PHAST and RPHAST: phylogenetic analysis with space/time models. Brief Bioinform 12: 41-51. doi:10 $.1093 / \mathrm{bib} / \mathrm{bbq072}$

Irimia M, Rukov JL, Penny D, Garcia-Fernandez J, Vinther J, Roy SW. 2008. Widespread evolutionary conservation of alternatively spliced exons in Caenorhabditis. Mol Biol Evol 25: 375-382. doi:10.1093/molbev/ $\mathrm{msm} 262$

Irimia M, Weatheritt RJ, Ellis JD, Parikshak NN, Gonatopoulos-Pournatzis T, Babor M, Quesnel-Vallières M, Tapial J, Raj B, O'Hanlon D, et al. 2014. A highly conserved program of neuronal microexons is misregulated in autistic brains. Cell 159: 1511-1523. doi:10.1016/j.cell.2014.11.035

Jaganathan K, Kyriazopoulou Panagiotopoulou S, McRae JF, Darbandi SF, Knowles D, Li YI, Kosmicki JA, Arbelaez J, Cui W, Schwartz GB, et al. 2019. Predicting splicing from primary sequence with deep learning. Cell 176: 535-548.e24. doi:10.1016/j.cell.2018.12.015

Kabat JL, Barberan-Soler S, McKenna P, Clawson H, Farrer T, Zahler AM. 2006. Intronic alternative splicing regulators identified by comparative genomics in nematodes. PLoS Comput Biol 2: e86. doi:10.1371/journal .pcbi.0020086

Kaletsky R, Lakhina V, Arey R, Williams A, Landis J, Ashraf J, Murphy CT. 2016. The $C$. elegans adult neuronal IIS/FOXO transcriptome reveals adult phenotype regulators. Nature 529: 92-96. doi:10.1038/ nature 16483

Kaletsky R, Yao V, Williams A, Runnels AM, Tadych A, Zhou S, Troyanskaya OG, Murphy CT. 2018. Transcriptome analysis of adult Caenorhabditis elegans cells reveals tissue-specific gene and isoform expression. PLoS Genet 14: e1007559. doi:10.1371/journal.pgen.1007559

Keren H, Lev-Maor G, Ast G. 2010. Alternative splicing and evolution: diversification, exon definition and function. Nat Rev Genet 11: 345-355. doi: $10.1038 / \mathrm{nrg} 2776$

Kim E, Magen A, Ast G. 2007. Different levels of alternative splicing among eukaryotes. Nucleic Acids Res 35: 125-131. doi:10.1093/nar/gkl924

Kotagama K, Schorr AL, Steber HS, Mangone M. 2019. ALG-1 influences accurate mrna splicing patterns in the Caenorhabditis elegans intestine and body muscle tissues by modulating splicing factor activities. Genetics 212: 931-951. doi:10.1534/genetics.119.302223

Kuroyanagi H, Ohno G, Mitani S, Hagiwara M. 2007. The Fox-1 family and SUP-12 coordinately regulate tissue-specific alternative splicing in vivo. Mol Cell Biol 27: 8612-8621. doi:10.1128/MCB.01508-07

Kuroyanagi H, Ohno G, Sakane H, Maruoka H, Hagiwara M. 2010. Visualization and genetic analysis of alternative splicing regulation in vivo using fluorescence reporters in transgenic Caenorhabditis elegans. Nat Protoc 5: 1495-1517. doi:10.1038/nprot.2010.107

Kuroyanagi H, Watanabe Y, Hagiwara M. 2013a. CELF family RNA-binding protein UNC-75 regulates Two sets of mutually exclusive exons of the unc-32 gene in neuron-specific manners in Caenorhabditis elegans. PLoS Genet 9: e1003337. doi:10.1371/journal.pgen.1003337

Kuroyanagi H, Watanabe Y, Suzuki Y, Hagiwara M. 2013b. Position-dependent and neuron-specific splicing regulation by the CELF family RNAbinding protein UNC-75 in Caenorhabditis elegans. Nucleic Acids Res 41: $4015-4025$. doi:10.1093/nar/gkt097

Lee Y, Rio DC. 2015. Mechanisms and regulation of alternative pre-mRNA splicing. Аппи Rev Biochem 84: 291-323. doi:10.1146/annurev-bio chem-060614-034316

Li YI, Sanchez-Pulido L, Haerty W, Ponting CP. 2015. RBFOX and PTBP1 proteins regulate the alternative splicing of micro-exons in human brain transcripts. Genome Res 25: 1-13. doi:10.1101/gr.181990.114

Li R, Ren X, Ding Q, Bi Y, Xie D, Zhao Z. 2020. Direct full-length RNA sequencing reveals unexpected transcriptome complexity during Caenorhabditis elegans development. Genome Res 30: 287-298. doi:10 $.1101 /$ gr.251512.119

Liao Y, Wang J, Jaehnig EJ, Shi Z, Zhang B. 2019. Webgestalt 2019: gene set analysis toolkit with revamped UIs and APIs. Nucleic Acids Res 47: W199-W205. doi:10.1093/nar/gkz401

Loria PM, Duke A, Rand JB, Hobert O. 2003. Two neuronal, nuclear-localized RNA binding proteins involved in synaptic transmission. Curr Biol 13: 1317-1323. doi:10.1016/S0960-9822(03)00532-3
Lyssenko NN, Hanna-Rose W, Schlegel RA. 2007. Cognate putative nuclear localization signal effects strong nuclear localization of a GFP reporter and facilitates gene expression studies in Caenorhabditis elegans. BioTechniques 43: 596-600. doi:10.2144/000112615

Merkin J, Russell C, Chen P, Burge CB. 2012. Evolutionary dynamics of gene and isoform regulation in mammalian tissues. Science 338: 1593-1599. doi:10.1126/science.1228186

Modrek B, Resch A, Grasso C, Lee C. 2001. Genome-wide detection of alternative splicing in expressed sequences of human genes. Nucleic Acids Res 29: 2850-2859. doi:10.1093/nar/29.13.2850

Norris AD, Calarco JA. 2012. Emerging roles of alternative pre-mRNA splicing regulation in neuronal development and function. Front Neurosci $\mathbf{6}$ : 122. doi:10.3389/fnins.2012.00122

Norris AD, Gao S, Norris ML, Ray D, Ramani AK, Fraser AG, Morris Q Hughes TR, Zhen M, Calarco JA. 2014. A pair of RNA-binding proteins controls networks of splicing events contributing to specialization of neural cell types. Mol Cell 54: 946-959. doi:10.1016/j.molcel.2014 .05 .004

Pan Q, Shai O, Misquitta C, Zhang W, Saltzman AL, Mohammad N, Babak T, Siu H, Hughes TR, Morris QD, et al. 2004. Revealing global regulatory features of mammalian alternative splicing using a quantitative microarray platform. Mol Cell 16: 929-941. doi:10.1016/j.molcel.2004.12.004

Pan Q Shai O, Lee LJ, Frey BJ, Blencowe BJ. 2008. Deep surveying of alternative splicing complexity in the human transcriptome by high-throughput sequencing. Nat Genet 40: 1413-1415. doi:10.1038/ng.259

Parras A, Anta H, Santos-Galindo M, Swarup V, Elorza A, Nieto-González JL, Picó S, Hernández IH, Díaz-Hernández JI, Belloc E, et al. 2018. Autismlike phenotype and risk gene mRNA deadenylation by CPEB4 mis-splicing. Nature 560: 441-446. doi:10.1038/s41586-018-0423-5

Pollard KS, Hubisz MJ, Rosenbloom KR, Siepel A. 2010. Detection of nonneutral substitution rates on mammalian phylogenies. Genome Res 20: 110-121. doi:10.1101/gr.097857.109

Quesnel-Vallières M, Dargaei Z, Irimia M, Gonatopoulos-Pournatzis T, Ip JY, Wu M, Sterne-Weiler T, Nakagawa S, Woodin MA, Blencowe BJ, et al. 2016. Misregulation of an activity-dependent splicing network as a common mechanism underlying autism spectrum disorders. Mol Cell 64: 1023-1034. doi:10.1016/j.molcel.2016.11.033

Ragle JM, Katzman S, Akers TF, Barberan-Soler S, Zahler AM. 2015. Coordinated tissue-specific regulation of adjacent alternative $3^{\prime}$ splice sites in C. elegans. Genome Res 25: 982-994. doi:10.1101/gr.186783.114

Raj B, Blencowe BJ. 2015. Alternative splicing in the mammalian nervous system: recent insights into mechanisms and functional roles. Neuron 87: 14-27. doi:10.1016/j.neuron.2015.05.004

Ramani AK, Calarco JA, Pan Q, Mavandadi S, Wang Y, Nelson AC, Lee LJ, Morris Q, Blencowe BJ, Zhen M, et al. 2011. Genome-wide analysis of alternative splicing in Caenorhabditis elegans. Genome Res 21: 342-348. doi:10.1101/gr.114645.110

Ray D, Kazan H, Cook KB, Weirauch MT, Najafabadi HS, Li X, Gueroussov S, Albu M, Zheng H, Yang A, et al. 2013. A compendium of RNA-binding motifs for decoding gene regulation. Nature 499: 172-177. doi:10.1038/ nature12311

R Core Team. 2018. R: a language and environment for statistical computing. $\mathrm{R}$ Foundation for Statistical Computing, Vienna. https://www.Rproject.org/.

Roach NP, Sadowski N, Alessi AF, Timp W, Taylor J, Kim JK. 2020. The fulllength transcriptome of C. elegans using direct RNA sequencing. Genome Res 30: 299-312. doi:10.1101/gr.251314.119

Romero PR, Zaidi S, Fang YY, Uversky VN, Radivojac P, Oldfield CJ, Cortese MS, Sickmeier M, LeGall T, Obradovic Z, et al. 2006. Alternative splicing in concert with protein intrinsic disorder enables increased functional diversity in multicellular organisms. Proc Natl Acad Sci 103: 83908395. doi:10.1073/pnas.0507916103

Saito Y, Yuan Y, Zucker-Scharff I, Fak JJ, Jereb S, Tajima Y, Licatalosi DD, Darnell RB. 2019. Differential NOVA2-mediated splicing in excitatory and inhibitory neurons regulates cortical development and cerebellar function. Neuron 101: 707-720.e5. doi:10.1016/j.neuron.2018.12.019

Scheckel C, Darnell RB. 2015. Microexons-tiny but mighty. EMBO J 34: 273-274. doi:10.15252/embj.201490651

Spencer WC, Zeller G, Watson JD, Henz SR, Watkins KL, McWhirter RD, Petersen S, Sreedharan VT, Widmer C, Jo J, et al. 2011. A spatial and temporal map of C. elegans gene expression. Genome Res 21: 325-341. doi:10 .1101 /gr.114595.110

Spencer WC, McWhirter R, Miller T, Strasbourger P, Thompson O, Hillier LDW, Waterston RH, Miller DM. 2014. Isolation of specific neurons from C. elegans larvae for gene expression profiling. PLoS One 9: e112102. doi:10.1371/journal.pone.0112102

Sugnet CW, Srinivasan K, Clark TA, O'Brien G, Cline MS, Wang H, Williams A, Kulp D, Blume JE, Haussler D, et al. 2006. Unusual intron conservation near tissue-regulated exons found by splicing microarrays. PLoS Comput Biol 2: e4. doi:10.1371/journal.pcbi.0020004 
Tapial J, Ha KCH, Sterne-Weiler T, Gohr A, Braunschweig U, HermosoPulido A, Quesnel-Vallières M, Permanyer J, Sodaei R, Marquez Y, et al. 2017. An atlas of alternative splicing profiles and functional associations reveals new regulatory programs and genes that simultaneously express multiple major isoforms. Genome Res 27: 1759-1768. doi:10 $.1101 /$ gr.220962.117

Torres-Méndez A, Bonnal S, Marquez Y, Roth J, Iglesias M, Permanyer J, Almudí I, O'Hanlon D, Guitart T, Soller M, et al. 2019. A novel protein domain in an ancestral splicing factor drove the evolution of neural microexons. Nat Ecol Evol 3: 691-701. doi:10.1038/s41559-019-0813-6

Tourasse NJ, Millet JRM, Dupuy D. 2017. Quantitative RNA-seq meta-analysis of alternative exon usage in C. elegans. Genome Res 27: 2120-2128. doi:10.1101/gr.224626.117

Ule J, Darnell RB. 2006. RNA binding proteins and the regulation of neuronal synaptic plasticity. Curr Opin Neurobiol 16: 102-110. doi:10.1016/j .conb.2006.01.003

Vaquero-Garcia J, Barrera A, Gazzara MR, González-Vallinas J, Lahens NF, Hogenesch JB, Lynch KW, Barash Y. 2016. A new view of transcriptome complexity and regulation through the lens of local splicing variations. eLife 5: e11752. doi:10.7554/eLife.11752

Vuong CK, Black DL, Zheng S. 2016. The neurogenetics of alternative splicing. Nat Rev Neurosci 17: 265-281. doi:10.1038/nrn.2016.27

Wamsley B, Jaglin XH, Favuzzi E, Quattrocolo G, Nigro MJ, Yusuf N, Khodadadi-Jamayran A, Rudy B, Fishell G. 2018. Rbfox1 mediates celltype-specific splicing in cortical interneurons. Neuron 100: 846859.e7. doi:10.1016/j.neuron.2018.09.026

Wang Z, Burge CB. 2008. Splicing regulation: from a parts list of regulatory elements to an integrated splicing code. RNA 14: 802-813. doi:10.1261/ rna.876308

Wang ET, Sandberg R, Luo S, Khrebtukova I, Zhang L, Mayr C, Kingsmore SF, Schroth GP, Burge CB. 2008. Alternative isoform regulation in human tissue transcriptomes. Nature 456: 470-476. doi:10.1038/nature07509

Wang Q, Abruzzi KC, Rosbash M, Rio DC. 2018. Striking circadian neuron diversity and cycling of drosophila alternative splicing. eLife 7: e35618. doi:10.7554/eLife.35618

Wani S, Kuroyanagi H. 2017. An emerging model organism Caenorhabditis elegans for alternative pre-mRNA processing in vivo. Wiley Interdiscip Rev RNA 8. doi:10.1002/wrna.1428

Warner AD, Gevirtzman L, Hillier LDW, Ewing B, Waterston RH. 2019. The C. elegans embryonic transcriptome with tissue, time, and alternative splicing resolution. Genome Res 29: 1036-1045. doi:10.1101/gr .243394 .118
White JG, Southgate E, Thomson JN, Brenner S. 1986. The structure of the nervous system of the nematode Caenorhabditis elegans. Philos Trans $R$ Soc London B, Biol Sci 314: 1-340. doi:10.1098/rstb.1986.0056

Wright PE, Dyson HJ. 2015. Intrinsically disordered proteins in cellular signalling and regulation. Nat Rev Mol Cell Biol 16: 18-29. doi:10.1038/ nrm3920

Xing Y, Lee CJ. 2005. Protein modularity of alternatively spliced exons is associated with tissue-specific regulation of alternative splicing. PLOS Genet 1: e34. doi:10.1371/journal.pgen.0010034

Xiong HY, Alipanahi B, Lee LJ, Bretschneider H, Merico D, Yuen RKC, Hua Y, Gueroussov S, Najafabadi HS, Hughes TR, et al. 2015. The human splicing code reveals new insights into the genetic determinants of disease. Science 347: 1254806. doi:10.1126/science.1254806

Xu Q, Modrek B, Lee C. 2002. Genome-wide detection of tissue-specific alternative splicing in the human transcriptome. Nucleic Acids Res 30: 3754-3766. doi:10.1093/nar/gkf492

Yang $X$, Coulombe-Huntington J, Kang S, Sheynkman GM, Hao T, Richardson A, Sun S, Yang F, Shen YA, Murray RR, et al. 2016. Widespread expansion of protein interaction capabilities by alternative splicing. Cell 164: 805-817. doi:10.1016/j.cell.2016.01.029

Yeo G, Holste D, Kreiman G, Burge CB. 2004. Variation in alternative splicing across human tissues. Genome Biol 5: R74. doi:10.1186/gb-2004-510-r74

Yeo GW, Van Nostrand E, Holste D, Poggio T, Burge CB. 2005. Identification and analysis of alternative splicing events conserved in human and mouse. Proc Natl Acad Sci 102: 2850-2855. doi:10.1073/pnas .0409742102

Yura K, Shionyu M, Hagino K, Hijikata A, Hirashima Y, Nakahara T, Eguchi T, Shinoda K, Yamaguchi A, Takahashi K-i, et al. 2006. Alternative splicing in human transcriptome: functional and structural influence on proteins. Gene 380: 63-71. doi:10.1016/j.gene.2006.05.015

Zahler AM. 2012. Pre-mRNA splicing and its regulation in Caenorhabditis elegans. WormBook 1-21. doi:10.1895/wormbook.1.31.2

Zhang C, Frias MA, Mele A, Ruggiu M, Eom T, Marney CB, Wang H Licatalosi DD, Fak JJ, Darnell RB. 2010. Integrative modeling defines the nova splicing-regulatory network and its combinatorial controls. Science 329: 439-443. doi:10.1126/science.1191150

Zheng S, Black DL. 2013. Alternative pre-mRNA splicing in neurons: growing up and extending its reach. Trends Genet 29: 442-448. doi:10.1016/j .tig.2013.04.003

Received June 12, 2020; accepted in revised form October 28, 2020.

\section{Genome Research}

www.genome.org 


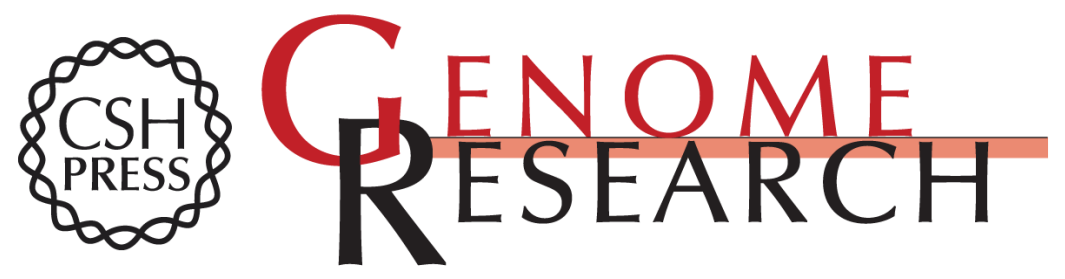

\section{Global regulatory features of alternative splicing across tissues and within the nervous system of $C$. elegans}

Bina Koterniak, Pallavi P. Pilaka, Xicotencatl Gracida, et al.

Genome Res. 2020 30: 1766-1780 originally published online October 30, 2020

Access the most recent version at doi:10.1101/gr.267328.120

Supplemental Material

References

Open Access

Creative Commons

License

Email Alerting Service
http://genome.cshlp.org/content/suppl/2020/11/16/gr.267328.120.DC1

This article cites 95 articles, 21 of which can be accessed free at: http://genome.cshlp.org/content/30/12/1766.full.html\#ref-list-1

Freely available online through the Genome Research Open Access option.

This article, published in Genome Research, is available under a Creative Commons License (Attribution 4.0 International), as described at http://creativecommons.org/licenses/by/4.0/.

Receive free email alerts when new articles cite this article - sign up in the box at the top right corner of the article or click here.

\section{Affordable, Accurate Sequencing.}

